ARTICLE

\title{
MDM2 E3 ligase-mediated ubiquitination and degradation of HDAC1 in vascular calcification
}

\author{
Duk-Hwa Kwon ${ }^{1, \star}$, Gwang Hyeon Eom ${ }^{1, \star}$, Jeong Hyeon Ko ${ }^{1}$, Sera Shin ${ }^{1}$, Hosouk Joung ${ }^{1}$, Nakwon Choe ${ }^{1}$, \\ Yoon Seok Nam¹, Hyun-Ki Min', Taewon Kook1, Somy Yoon', Wanseok Kang ${ }^{2}$, Yong Sook Kim², \\ Hyung Seok Kim ${ }^{3}$, Hyuck Choi ${ }^{4}$, Jeong-Tae Koh ${ }^{4}$, Nacksung Kim¹, Youngkeun Ahn ${ }^{2}$, Hyun-Jai Cho ${ }^{5}$, \\ In-Kyu Lee ${ }^{6}$, Dong Ho Park ${ }^{7}$, Kyoungho Suk ${ }^{8}$, Sang Beom Seo ${ }^{9}$, Erin R. Wissing ${ }^{10}$, Susan M. Mendrysa ${ }^{10}$, \\ Kwang-II Nam ${ }^{11} \&$ Hyun Kook ${ }^{1}$
}

Vascular calcification (VC) is often associated with cardiovascular and metabolic diseases. However, the molecular mechanisms linking VC to these diseases have yet to be elucidated. Here we report that MDM2-induced ubiquitination of histone deacetylase 1 (HDAC1) mediates VC. Loss of HDAC1 activity via either chemical inhibitor or genetic ablation enhances VC. HDAC1 protein, but not mRNA, is reduced in cell and animal calcification models and in human calcified coronary artery. Under calcification-inducing conditions, proteasomal degradation of HDAC1 precedes VC and it is mediated by MDM2 E3 ubiquitin ligase that initiates HDAC1 K74 ubiquitination. Overexpression of MDM2 enhances VC, whereas loss of MDM2 blunts it. Decoy peptide spanning HDAC1 K74 and RG 7112, an MDM2 inhibitor, prevent $V C$ in vivo and in vitro. These results uncover a previously unappreciated ubiquitination pathway and suggest MDM2-mediated HDAC1 ubiquitination as a new therapeutic target in VC.

\footnotetext{
${ }^{1}$ Department of Pharmacology and Medical Research Center for Gene Regulation, Chonnam National University Medical School, 5 Hak-dong, Dong-ku, Gwangju 501-746, Republic of Korea. ${ }^{2}$ Department of Cardiology, Chonnam University Hospital, Gwangju 501-757, Republic of Korea. ${ }^{3}$ Department of Forensic Medicine, Chonnam National University Medical School, 5 Hak-dong, Dong-ku, Gwangju 501-746, Republic of Korea. ${ }^{4}$ Department of Pharmacology and Dental Therapeutics, Research Center for Biomineralization Disorders, School of Dentistry, Chonnam National University, Gwangju 500-747, Republic of Korea. ${ }^{5}$ Division of Cardiology, Department of Internal Medicine, Seoul National University Hospital, Seoul 110-744, Republic of Korea. ${ }^{6}$ Department of Internal Medicine, Kyungpook National University School of Medicine, Daegu 700-721, Republic of Korea. ${ }^{7}$ Department of Ophthalmology, Kyungpook National University School of Medicine, Daegu 700-721, Republic of Korea. ${ }^{8}$ Department of Pharmacology, Brain Science \& Engineering Institute, Kyungpook National University School of Medicine, Daegu 700-721, Republic of Korea. ${ }^{9}$ Department of Life Science, Chung-Ang University, Seoul 156-756, Republic of Korea. ${ }^{10}$ Department of Basic Medical Sciences, College of Veterinary Medicine, Purdue University, West Lafayette, IN 47907, USA. ${ }^{11}$ Departments of Anatomy, Chonnam National University Medical School, 5 Hak-dong, Dong-ku, Gwangju 501-746, Republic of Korea. * These authors contributed equally to this work. Correspondence and requests for materials should be addressed to H.K. (email: kookhyun@chonnam.ac.kr).
} 
$\mathrm{V}$ ascular calcification (VC), a deposition of calcium phosphate in arteries, heart valves and cardiac muscle, often results in the development of atherosclerotic intimal injury and in a rapid rise of stiffness of blood vessels. As a concomitant adverse effect of diabetes and chronic renal failure, VC is often the cause of high morbidity and mortality associated with these chronic diseases ${ }^{1}$. Indeed, VC is closely related to the increase in risk of myocardial infarction and dissection after angioplasty $^{2}$. Despite its clinical implications, the molecular mechanisms of VC have yet to be elucidated. Recent advances utilizing genetically modified mice have shown that VC is an active process rather than a degenerative, passive process at the end stage of vascular diseases ${ }^{3}$. For example, knockout (KO) of matrix Gla protein ${ }^{4}$, KLOTHO ${ }^{5,6}$, carbonic anhydrase $\mathrm{II}^{7}$, desmin $^{8}$ or osteoprotegerin ${ }^{9}$ in mice elicits marked enhancement of VC, which suggests that VC is in fact actively regulated by vascular smooth muscle acquiring osteoblast-like properties.

Many researchers have reported that histone deacetylase (HDAC) inhibitors (HDACi) are beneficial in regression of tumour proliferation $^{10}$ and in blockade of fibrosis ${ }^{11,12}$. In cardiac diseases, HDACi inhibit arrhythmia ${ }^{13}$ and myocardial infarction ${ }^{14,15}$. Many research groups, including ours 16,17 , have shown that HDACi also prevent cardiac hypertrophy ${ }^{17,18}$. Thus, we had first postulated that HDACi would have preventive effects in VC. On the contrary, however, we found that HDAC inhibition resulted in the enhancement of VC. We therefore further investigated the functional roles of HDACs in the development of VC. Here we report that proteasome-dependent degradation of HDAC1 results in the enhancement of $\mathrm{VC}$, and that MDM2 acts as an E3 ubiquitin ligase of $\mathrm{HDAC1}$ in response to calcification stimuli.

\section{Results}

Class I HDAC inhibition enhances VC. Treatment of primary cultured rat vascular smooth muscle cells (RVSMCs) with trichostatin A (TSA), a non-class-selective HDACi, did not affect calcification. However, it significantly enhanced inorganic phosphate ( $\mathrm{Pi}, 2 \mathrm{mM}$ )-induced calcium deposition in RVSMCs (Supplementary Fig. 1a), which was quantified with calcium content measurement (Supplementary Fig. 1b). We further studied whether this enhancement of calcium deposition would be repeated with the class-I-selective $\mathrm{HDACi}$ apicidin $^{18}$. Although apicidin itself did not induce VC, it potentiated Pi-induced calcium deposition (Fig. 1a,b). Non-class-selective HDACimediated enhancement of VC was also recently reported in a cellular model, and an increase in osteogenic Runx2 was elucidated as a possible mechanism ${ }^{19}$. Indeed, in our experimental models, we observed that apicidin, as well as TSA enhanced the expression of Runx2 (Fig. 1c), which had been known to play a key role in the development of $\mathrm{VC}^{20}$. HDACi $(10 \mathrm{nM}$ TSA and $50 \mathrm{nM}$ apicidin) and $\mathrm{Pi}(2 \mathrm{mM})$ did not affect RVSMC survival as determined by 3-(4,5-dimethylthiazol-2-yl)2,5-diphenyltetrazolium bromide (MTT) assay (Supplementary Fig. 1c). In an ex vivo experimental model in which whole isolated aorta was cultured in DMEM, Pi induced calcium deposition in the aorta, which was then significantly enhanced by either TSA or apicidin (Fig. 1d). We next sought to determine whether inhibition of HDAC potentiates VC in mice. Vitamin $\mathrm{D}_{3}\left(\mathrm{VD}_{3}\right)$ injection caused hypercalcemia (Supplementary Fig. 1d). $\mathrm{VD}_{3}$-induced VC was enhanced by TSA (Fig. 1e), although the serum calcium level was not changed (Supplementary Fig. 1d). Quantification of calcium content clearly showed that administration of TSA enhanced $\mathrm{VD}_{3}$-induced VC (Fig. 1f).

Loss of HDAC1 potentiates VC. Next we questioned which HDAC among the class I HDACs (HDAC1, 2, 3 and 8) is responsible for the potentiation of VC. HDAC1 short interfering RNA (siRNA) reiterated the effect of the HDACi (Fig. 2a,b; Supplementary Fig. 1e). Adenoviral overexpression of HDAC1 (Ad-HDAC1) caused a decrease in calcium content (Fig. 2c; Supplementary Fig. 1f), but HDAC2 siRNA did not affect VC (Fig. 2d; Supplementary Fig. 1g). In contrast, forced expression of HDAC1 with Ad-HDAC1 reduced the expression of Runx2 (Fig. 2e). The involvement of HDAC1 was further confirmed in HDAC1 KO mice. Because conventional genetic disruption of HDAC1 in the whole body results in embryonic lethality ${ }^{21}$, we generated vascular smooth muscle-specific $\mathrm{KO}$ of $\mathrm{HDAC1}$ (HDAC1-cKO; Supplementary Fig. 1h). $\mathrm{VD}_{3}$-induced calcification was enhanced in HDAC1-cKO mice (Fig. 2f). A transverse section of the aorta showed that the calcification area was expanded in $\mathrm{VD}_{3}$-treated $\mathrm{HDAC1}$-cKO mice (Fig. 2g), which resulted in the enhancement of calcium deposition in the proximal aorta (Fig. 2h). VC was prominent in the proximal aorta and its branches when observed by three-dimensional reconstruction after computed tomography (Fig. 2i, arrowheads). Serum calcium level was not altered in HDAC1-cKO (Supplementary Fig. 1i).

HDAC1 protein amounts are reduced in calcification condition. Among class I HDACs, HDAC1 and HDAC2 were significantly reduced by Pi in RVSMCs (Fig. 3a). The reductions of HDAC1 and HDAC2 protein amounts were quantified (Supplementary Fig. 2a,b) and the reduction of HDAC1 was greater than that of HDAC2. By time-course analysis, we found that the reduction of HDACl (Supplementary Fig. 2c) preceded the substantial increase in calcium deposition that was peaked 5-6 days after $\mathrm{Pi}$ treatment (Fig. 3b), which suggests that reduction of HDAC1 is a cause of VC. We also observed that Pi-induced calcium deposition (Supplementary Fig. 2d) causes the reduction of protein amount of HDAC1 (Supplementary Fig. 2e) in human coronary artery smooth muscle cells (HCASMCs).

In other in vitro calcification models, osteogenic medium ${ }^{22}$ (OM) induced VC (Supplementary Fig. 2f), which also caused a reduction of HDAC1 (Supplementary Fig. $2 \mathrm{~g}$ ). $\mathrm{CaCl}_{2}(8 \mathrm{mM})$, but not $\beta$-glycerophosphate $(10 \mathrm{mM})$, significantly reduced HDAC1 amounts (Supplementary Fig. 2h). Phosphonoformic acid, a Pi transporter inhibitor ${ }^{23}$, completely blocked VC (Supplementary Fig. 2i), which resulted in recovery of the reduced HDAC1 protein amounts (Supplementary Fig. 2j). Next, we questioned whether the decrease in the protein amounts resulted from a decrease in transcription of HDAC1 or an increase in protein degradation. Interestingly, transcript levels of all class I HDACs were increased by $\mathrm{Pi}$, whereas class II HDACs were not altered (Fig. 3c; Supplementary Fig. 2k). When de novo synthesis of protein was blocked by cycloheximide, HDACl decay was significantly accelerated (Fig. 3d).

In $\mathrm{VD}_{3}$-treated mice, the protein amounts of HDAC1 were significantly reduced in aorta in a dose-dependent manner (Fig. 3e). The reduction of HDAC1 was confirmed by histologic evaluation (Supplementary Fig. 3a), and was further quantified (Supplementary Fig. 3b). In contrast, we were not able to observe any significant reduction of messenger RNA (mRNA) levels (Supplementary Fig. 3c,d).

Clinically, atherosclerosis is closely related to the development of intimal $\mathrm{VC}^{24}$. Thus, we examined whether HDAC1 expression was also reduced in the atherosclerosis-associated VC models. As described previously ${ }^{25}$, a diet high in cholesterol plus calcium markedly induced calcification (Fig. $3 \mathrm{f}$ ), as well as atherosclerosis in ApoE KO mice. In the localized area where the severe calcification developed, HDAC1 expression was decreased (Fig. 3f, arrowheads). VC is often associated with atherosclerosis 

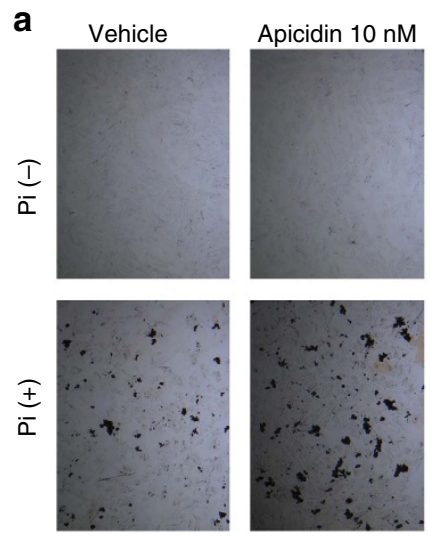

RVSMCs

C

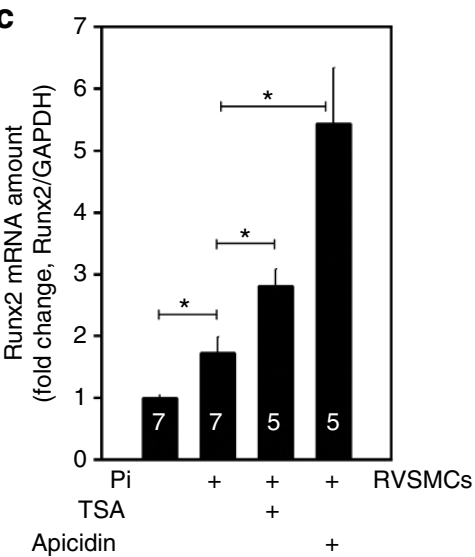

e

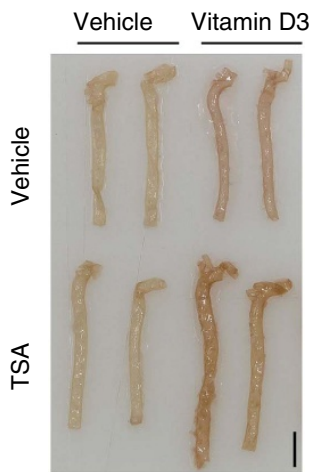

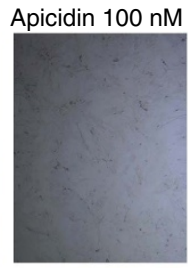

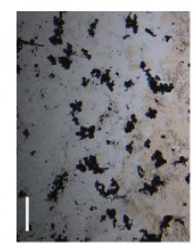

d
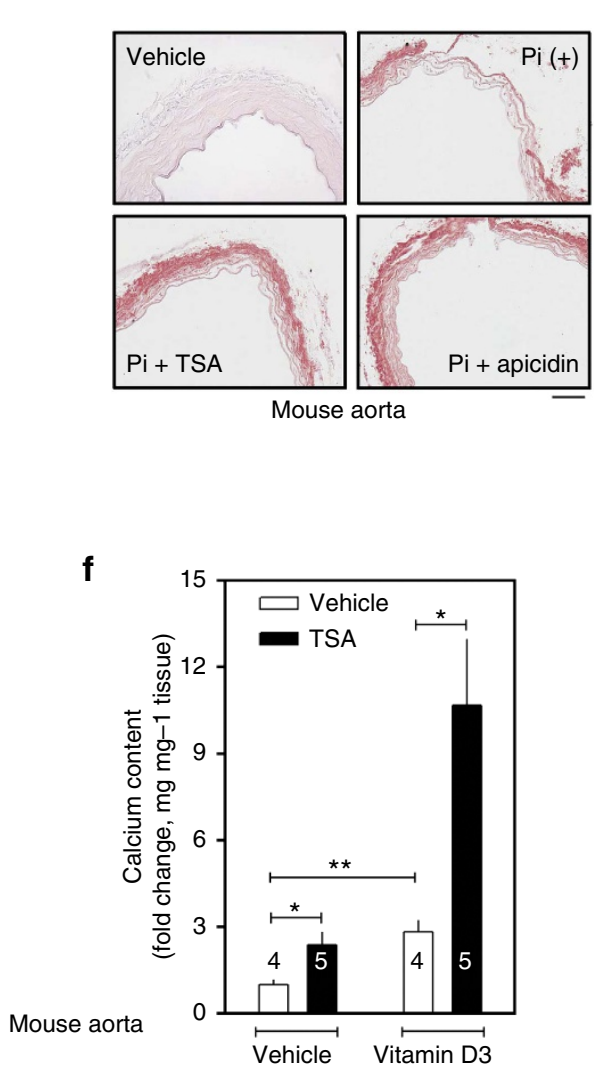

Figure 1 | HDAC inhibitors potentiate vascular calcification (VC). (a) Apicidin, a class-I-selective HDACi, potentiated the Pi-induced VC in a dose-dependent manner. VC was induced either by inorganic phosphate $(\mathrm{Pi})$ in rat vascular smooth muscle cells (RVSMCs) or by vitamin $\mathrm{D}_{3}\left(\mathrm{VD} \mathrm{D}_{3}\right)$ in mouse. Scale bar, $100 \mu \mathrm{m}$. (b) Quantification results. Samples (8-10)were measured from three independent experimental sets. (c) Both $10 \mathrm{nM}$ TSA and $50 \mathrm{nM}$ apicidin potentiated Pi-induced induction of Runx2. Quantitative real-time RT-PCR was performed. Each sample was measured in duplicate and counted as one case ( $n=5-7$ from two sets). (d) Induction of calcification of aorta ex vivo revealed enhancement of VC by Pi. Alizarin red S staining. Scale bar, $100 \mu \mathrm{m}$. (e) TSA $\left(0.6 \mathrm{mg} \mathrm{kg}^{-1}\right.$, intraperitoneally for 9 days) potentiated $V C$ induced by $V_{3}\left(5 \times 10^{5} \mathrm{IU} \mathrm{kg}^{-1}\right.$ per day, subcutaneous administered for the first 3 days). Calcification was determined with Alizarin red $\mathrm{S}$ staining. Scale bar, $3 \mathrm{~mm}$. (f) Quantification results of calcium content in the proximal aorta. Calcium contents from four to five mice in one experimental set were measured. Error bars represent s.e.m. ${ }^{\star} P<0.05$, ${ }^{\star \star} P<0.01$, Numerals in bar graphs are the numbers of samples.

even without apparent calcification stimuli $^{26}$. Thus, we investigated whether HDAC1 was also reduced in atherosclerosisassociated calcification without administration of $\mathrm{VD}_{3}$ or calcium. Carotid artery ligation in $A p o E \mathrm{KO}$ mice significantly induced atheromatous plaques as described previously ${ }^{27}$. Some of the mice ( $\sim 30 \%)$ developed localized calcification (arrowheads in lower left panel in Fig. 3g), regardless of the severity of atheroma, and HDAC1 expression was downregulated (lower right panel in
Fig. 3g) in the ligated mice compared with the non-ligation group (upper right panel). Quantification of the histological analysis is provided in Supplementary Fig. 3e. For further evaluation in a human disease, calcified human coronary artery samples were used for HDAC1 immunohistochemistry. Compared with the expression in coronary artery from normal patients, severe calcification reduced the expression of HDAC1 (Fig. 3h). The changes in the expression of HDAC1 are quantified in 
a

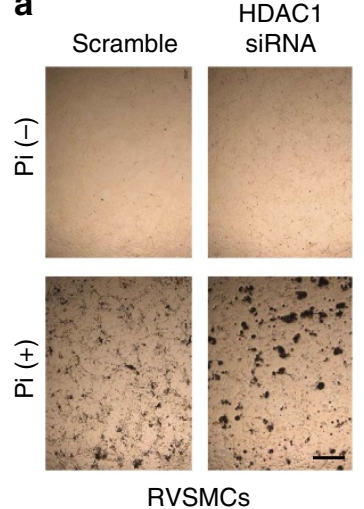

f

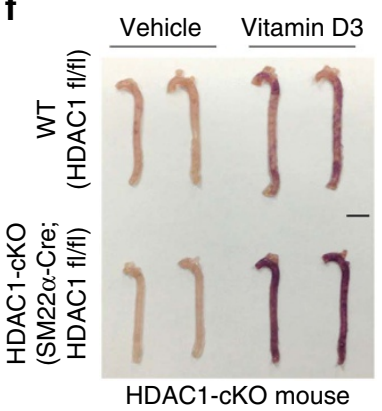

b

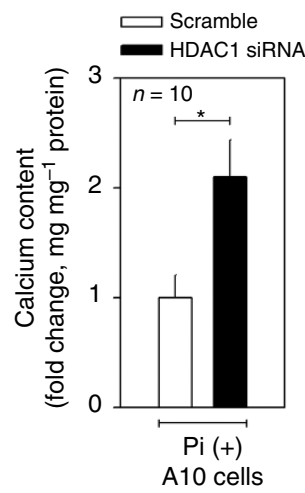

g

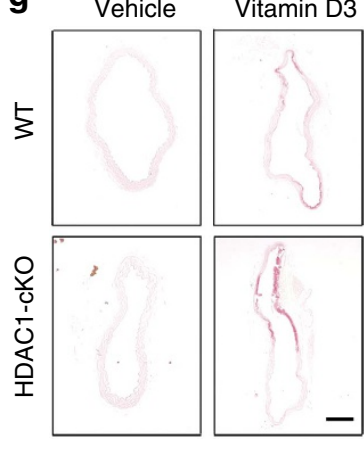

HDAC1-cKO mouse
C

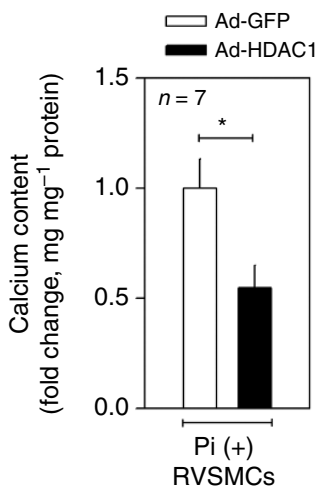

d

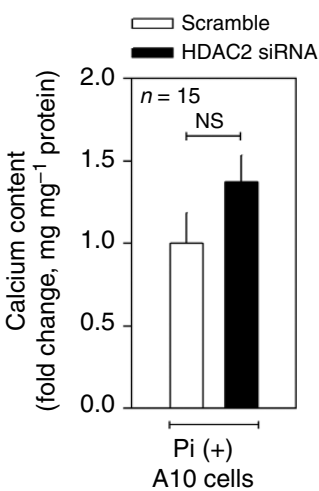

e

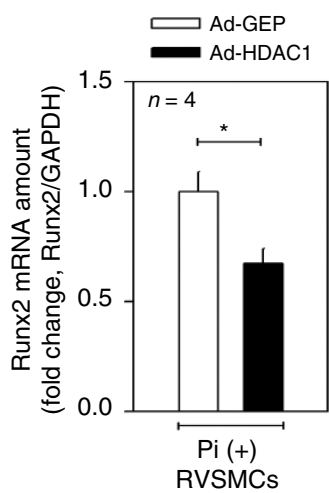

h

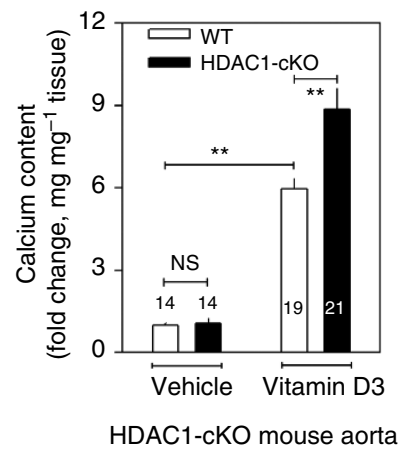

i

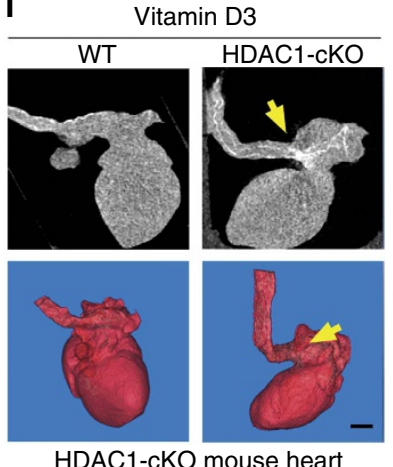

Figure 2 | Loss of HDAC1 enhances vascular calcification (VC). (a) Transfection of HDAC1 small interfering RNA (siRNA, $25 \mathrm{nM)} \mathrm{potentiated} \mathrm{Pi-induced}$ VC. Von Kossa staining. Scale bar, $100 \mu \mathrm{m}$. (b) Quantification results of calcium content in HDAC1 siRNA-transfected A10 cells. Ten samples from three independent sets were measured. (c) Infection of adenoviral HDAC1 (Ad-HDAC1) to RVSMCs blunted Pi-induced VC. RVSMCs were treated with Ad-HDAC1 $(50 \mathrm{MOI})$, kept in serum-free condition for $24 \mathrm{~h}$ and then switched to Pi-containing media. Cells were then treated with Ad-HDAC1 every 2 days ( $n=7$ from two sets). (d) Reduction of HDAC2 by HDAC2 siRNA ( $25 \mathrm{nM}$ ) did not potentiate Pi-induced VC ( $n=15$ samples from three sets). (e) Ad-HDAC1 reduced Runx2 mRNA amount. Each sample was measured in duplicate and counted as one case ( $n=4$ from two sets). (f) Vascular smooth

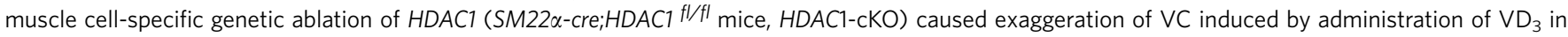
mice, compared with $H D A C 1{ }^{f l / f l}$ control. $V D_{3}$ was administered to 6-8-week-old HDAC1-cKO or HDAC1 ${ }^{f l / f l}$ male mice. Alizarin red $S$ staining. Scale bar, $3 \mathrm{~mm}$. (g) Horizontal sections of aorta showing VC in HDAC1-cKO mice. Scale bar, $200 \mu \mathrm{m}$. (h) Quantification results of calcium deposition in HDAC1-cKO mouse aorta. (i) Computed tomography (CT) images showing enhanced calcification in the arch of aorta. Arrows indicate the calcification foci at the proximal aorta and its branches. Upper panels: CT images. Lower panels: three-dimensional reconstruction images. Scale bar, $1 \mathrm{~mm}$. Error bars represent s.e.m. ${ }^{\star} P<0.05,{ }^{\star \star} P<0.01, \mathrm{NS}$, not significant. Numerals in bar graphs are the numbers of samples.

Supplementary Fig. 3f. The mRNA level of HDAC1, however, was not altered in calcified human coronary artery compared with normal (Supplementary Fig. 3g). These results suggest that reduction of HDAC1 protein, but not mRNA, is associated with VC.

HDAC1 is degraded through $\mathrm{K} 74$ ubiquitination. We next studied the mechanism of the degradation of HDAC1 in VC. As shown in Fig. 4a, Pi-induced reduction of HDAC1 was markedly attenuated by treatment with MG132, a proteasome inhibitor, but not by treatment with inhibitors of lysosome (chloroquine) or autophagy (3-methyladenine). Alternative proteasome inhibitors also prevented HDAC1 degradation (Supplementary Fig. 4a). By the use of immunoprecipitation-based ubiquitination assays, we found that in the presence of MG132 and ubiquitin, Flag-tagged exogenous HDAC1 was ubiquitinated in A10 cells, a RVSMC line (4th lane in Fig. 4b, immunoprecipitation with ubiquitin (anti-HA antibody) and immunoblotting with HDAC1 (anti-HDAC1 antibody)). Interestingly, the ubiquitination of HDAC1 was further enhanced by Pi (5th lane in Fig. 4b).

To further rule out non-proteolytic ubiquitination of HDAC1, a tandem ubiquitin-binding entities (TUBEs) ${ }^{28,29}$ assay was performed. The TUBE assay recognizes four sequential conjugations of ubiquitin molecules (tetra-ubiquitin), that is, ubiquitination. With Pi-treated A10 cells, a pull-down assay was performed with either glutathione $S$-transferase (GST) or GST-TUBE, and then the precipitates were subjected to immunoblotting with anti-HDAC1 antibody. The TUBE assay showed that HDAC1 ubiquitination is increased by Pi treatment in A10 cells (Fig. 4c). When RVSMCs were treated with MG132 and lactacystin, an alternative proteasome inhibitor, the amount of calcium deposition induced by Pi was halved (Fig. 4d,e). The effect of proteasome inhibitor was also investigated in vivo; intraperitoneal administration of MG132 significantly reduced $\mathrm{VD}_{3}$-induced calcium deposition (Fig. 4f,g) and prevented the reduction of HDAC1 expression (Supplementary Fig. 4b), whereas it did not affect the serum calcium level (Supplementary Fig. 4c).

Next, we isolated the lysine residues responsible for the HDAC1 ubiquitination. Using bioinformatics analysis (www.phosphosite.org), we investigated K74 and K89, which are well conserved (Supplementary Fig. 4d). We generated two mutant mammalian expression vectors of HDAC1 (K74R and K89R) and performed GST pull-down-based TUBE assay in A10 
a

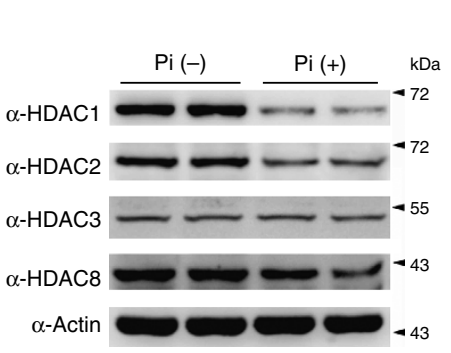

RVSMCs b

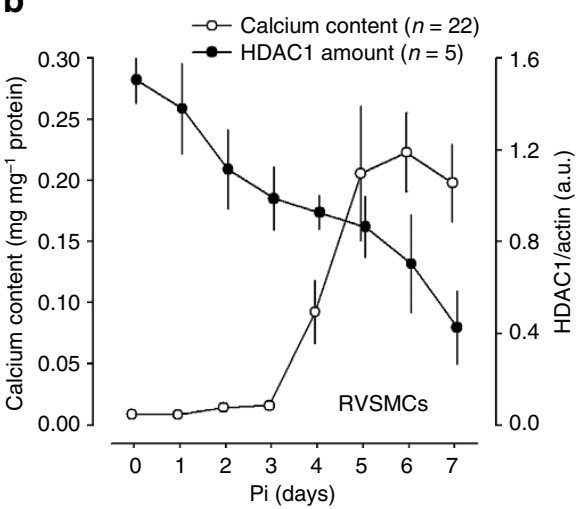

C

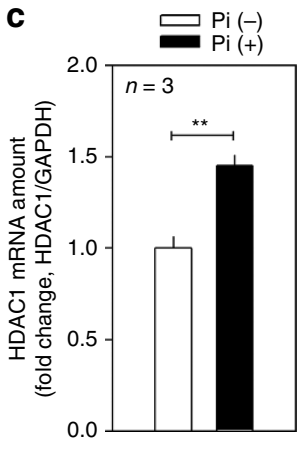

d

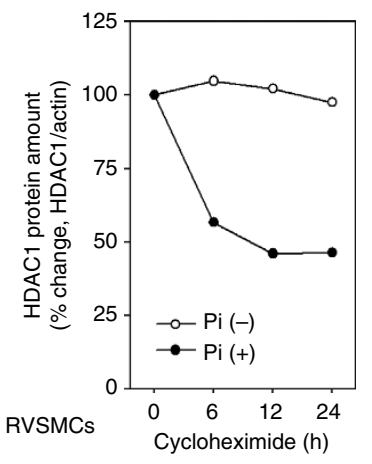

e

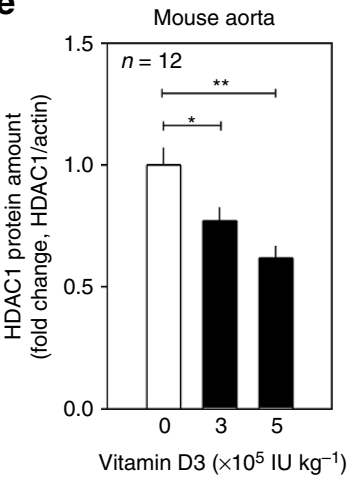

f

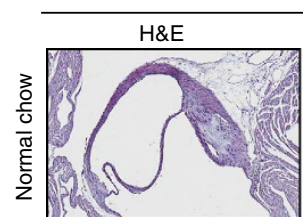

ApoE KO mice + cholesterol / calcium diet Alizarin red $\mathrm{S}$
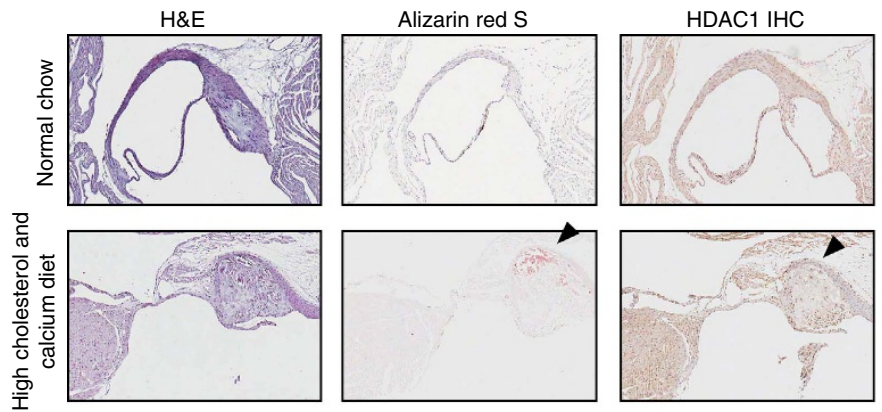
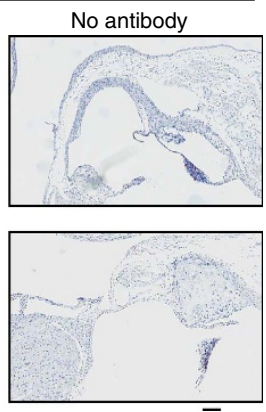

g
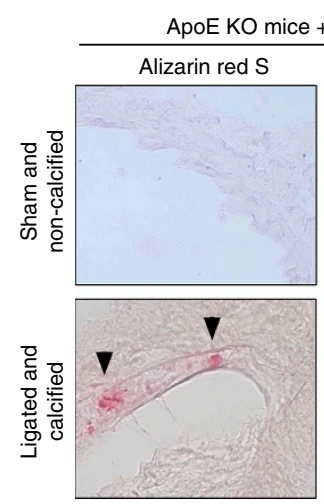

$\mathbf{h}$
Human coronary artery - intimal calcification (atherosclerosis)

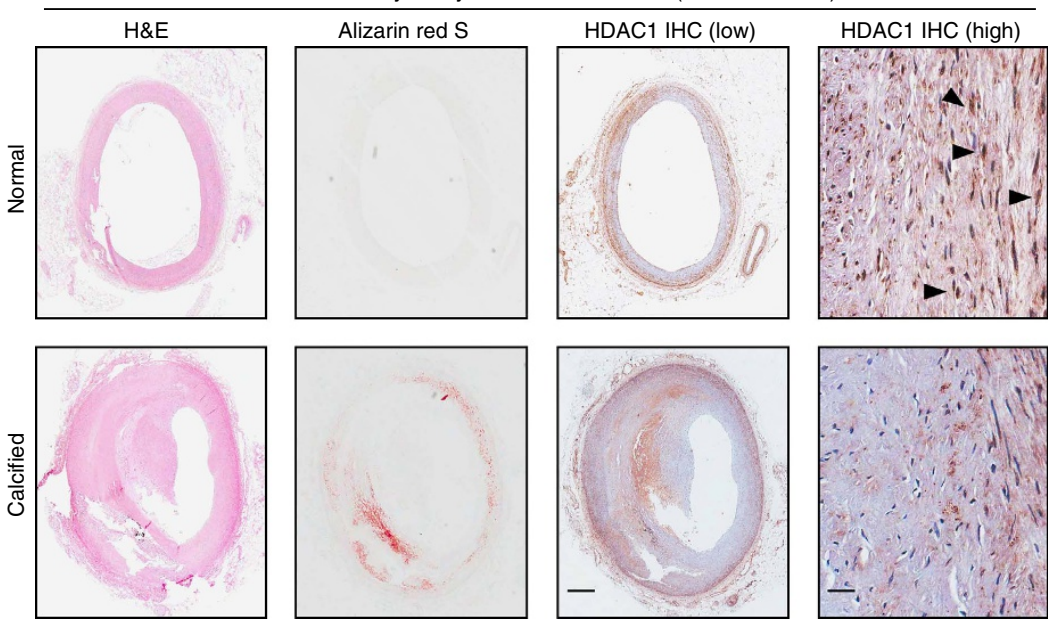

Figure 3 | HDAC1 protein, but not mRNA, is reduced in VC. (a) Among the class I HDACs, HDAC1 and HDAC2 protein amounts were reduced by Pi treatment. (b) Time course of calcium deposition (open circle) and HDAC1 protein reduction (black circle). Note that HDAC1 protein reduction precedes substantial increase in calcium content. Calcium contents were measured in 22 samples from six sets and quantification results of HDAC1 protein amounts were obtained from five samples out of three independent sets of experiments. (c) Changes in HDAC1 mRNA levels. mRNA content was determined with quantitative real-time RT-PCR. Each sample was measured in duplicate and counted as one case $(n=3$ from one set). (d) Cycloheximide (CHX) chase study elicited enhancement of HDAC1 protein decay by Pi. After treatment with Pi ( $2 \mathrm{mM}, 6$ days), $20 \mu \mathrm{g} \mathrm{ml} \mathrm{I}^{-1} \mathrm{CHX}$ was treated for the indicated interval. Values were averaged from two experiments. (e) $V D_{3}$ significantly reduced the protein amount of HDAC1 in the aorta in a dose-dependent manner. Quantification results from 12 western blots from four independent experimental sets. (f) Immunohistochemical analysis showed the reduction of HDAC1 in atherosclerosis-associated calcification mouse models. Ten-week-old ApoE knockout (KO) male mice were fed a high-cholesterol diet for 10 weeks followed by a high-cholesterol plus calcium diet for the next 7 weeks. The HDAC1 expression level was downregulated in the tissues adjacent to the calcified focus (arrowheads). Scale bar, $100 \mu \mathrm{m}$. (g) Immunohistochemical analysis showed a reduction of HDAC1 in an alternative atherosclerosis animal model. ApoE KO male mouse aorta was subjected to carotid artery ligation to induce sheer stress and atherosclerosis developed. Some mice showed calcified foci (arrowheads) adjacent to the atherosclerotic plaque where HDAC1 expression was lowered. Scale bar, $25 \mu \mathrm{m}$. (h) HDAC1 protein level was downregulated in atherosclerosis-associated human coronary artery. Arrowheads indicate HDAC1-positive nuclei. Scale bar, $500 \mu \mathrm{m}$ (low power); $25 \mu \mathrm{m}$ (high power). ${ }^{\star} P<0.05,{ }^{\star \star} P<0.01$. 
cells transfected with either HDAC1 wild-type or HDAC1 mutants in the presence of MG132 and $\mathrm{Pi}$ stimulation. The results showed that HDAC1 ubiquitination was blunted in HDAC1 K74R, whereas HDAC1 K89R ubiquitination was not attenuated (Fig. 4h). Indeed, in the absence of MG132, Pi-induced reduction of HDAC1 protein was blunted in HDAC1 K74R-transfected A10 cells (Supplementary Fig. 4e).

We further questioned whether specific blockade of HDAC1 ubiquitination might prevent VC. We designed a 15 amino-acidlong synthetic peptide spanning the K74 residue. This peptide was linked with fluorescein isothiocyanate-conjugated nuclear localization signal (NLS) peptide ${ }^{30}$ (Supplementary Fig. 4f). Compared with scramble peptide conjugated with fluorescein isothiocyanate, the synthetic peptide successfully incorporated in the nucleus in RVSMCs (Supplementary Fig. 4g). K74 peptide successfully blocked the Pi-induced reduction of the HDAC1 protein amount (Fig. 4i) and attenuated calcium deposition in RVSMCs (Fig. 4j). These results suggested that the synthetic peptide spanning K74 worked as a decoy peptide to prevent VC in RVSMCs.

MDM2 induces ubiquitination of HDAC1 in VSMCs. E3 ligases often determine the specificity of ubiquitin proteasome protein degradation. To find the specific E3 ligase for HDAC1 degradation in VC, we postulated that the E3 ligase might (1) be upregulated in VC, (2) induce calcification and (3) ubiquitinate HDAC1 in RVSMCs. We first performed complementary DNA (cDNA) microarray with Pi-treated RVSMCs (Supplementary Fig. 5a,b). Seven E3 ligases were included in the dysregulated genes. Among those E3 ligases, two F-box proteins (FBXO4 and FBXO32) and MDM2 were upregulated by Pi (Supplementary Fig. $5 \mathrm{c}$ ). We further confirmed the changes in amounts of those 7 E3 ligases by quantitative PCR with reverse transcription (qRT-PCR) and found that MDM2 was most upregulated (Fig. 5a). We checked the transcript levels of four different E3 ligases previously reported in the literature to ubiquitinate HDAC1: $C H F R^{31}$, MDM2 (ref. 32), PIRH2 (ref. 33) and $R E N^{34}$. Only $M D M 2$ was significantly increased by $\mathrm{Pi}$ treatment in RVSMCs (Fig. 5b). Pi treatment for 3 or 6 days also increased the protein amount of MDM2 in RVSMCs (Fig. 5c; Supplementary Fig. 6a). We measured changes in the protein amounts of the other four E3 ligases (ATG3, FBXO4, FBXO32 and SIAH2), the mRNA amounts of which were significantly increased among cDNA microarray-based candidates; none of the other E3 ligases were significantly altered at the protein level (Supplementary Fig. 6b).

Next, we checked whether HDAC1 and MDM2 could physically interact. The immunoprecipitation assay showed that exogenous HDAC1 physically interacted with exogenous MDM2 in 293T cells (Supplementary Fig. 6c,d). The association of both proteins was further observed with endogenous proteins in RVSMCs (Fig. 5d). To confirm the direct interaction between MDM2 and HDAC1, we utilized the GST pull-down assay in the cell-free condition. Bacterially overexpressed His-MDM2 protein was successfully pulled down by Sepharose 4B-bound GSTHDAC1 (second lane in the upper gel in Fig. 5e), which suggests that no other protein is involved in the interaction. Infection of adeno-MDM2 (Ad-MDM2) reduced exogenous HDAC1 protein expression by Ad-HDAC1 in a dose-dependent manner in RVSMCs (Fig. 5f). Transient transfection of HA-MDM2 also potentiated Pi-induced calcium deposition in A10 cells (Supplementary Fig. 6e).

MDM2 possesses a RING domain that is indispensable for ubiquitination activity ${ }^{35}$. Deletion of the RING domain of MDM2 $(\mathrm{MDM} 2 \Delta \mathrm{R})$ results in the loss of E3 ligase activity ${ }^{36}$. Compared with wild-type MDM2, which induced ubiquitination of HDAC1, MDM2 $\Delta \mathrm{R}$ failed to do so (Fig. 5g). MDM2 failed to induce ubiquitination of HDAC1 K74R (Fig. 5h), but did induce ubiquitination of K89 (Supplementary Fig. 6f). These results suggest that $\mathrm{Pi}$ induces MDM2 and the increase in MDM2 results in the ubiquitination of HDAC1 K74 in RVSMCs.

MDM2 induces calcium deposition in a p53-independent manner. Next, we questioned whether MDM2 can induce VC. Infection of Ad-MDM2 induced calcium deposition in RVSMCs only at a higher dose (fourth column in Fig. 6a). However, under Pi treatment, Ad-MDM2 significantly potentiated the calcium deposition in a dose-dependent manner in RVSMCs (fifth to eighth columns in Fig. 6a). In addition, MDM2 siRNA (Supplementary Fig. 7a) significantly attenuated Pi-induced calcium deposition in A10 cells (Fig. 6b).

[(4S,5R)-2-(4-tert-butyl-2-ethoxyphenyl)-4,5-bis(4-chlorophenyl)4,5-dimethylimidazol-1-yl]-[4-(3-methylsulfonylpropyl)piperazin1-yl]methanone (RG 7112, (RG)), a member of the Nutlin family, has been recently developed as a novel inhibitor of MDM2. RG stabilizes p53 to elicit anticancer activity ${ }^{37}$. As a mechanism, it has been reported that RG interferes with the association of MDM2 with p53 by masking the binding surface of MDM2 (ref. 38). Thus, we checked whether RG could also block MDM2 activity by interfering with binding to HDAC1 and thereby whether RG could prevent VC. Treatment with RG $(2.5 \mu \mathrm{M})$ resulted in the dissociation of HDAC1 and MDM2 (Fig. 6c). RG $(0.1 \mu \mathrm{M})$ prevented the $\mathrm{Pi}$-induced reduction of HDAC1 in A10 cells (Fig. 6d). Von Kossa staining revealed that RG blocked calcium deposition in a dose-dependent manner in RVSMCs (Fig. 6e). RG $(0.5 \mu \mathrm{M})$ blocked VC in RVSMCs (Fig. 6f). In HCASMCs, calcium deposition by Pi treatment for either 3 or 6 days was significantly attenuated in RG in a dose-dependent manner (Fig. 6g).

\footnotetext{
Figure 4 | HDAC1 is ubiquitinated in VC. (a) MG132 (10 $\mu \mathrm{M}$, proteasome inhibitor) but not chloroquine (100 $\mu \mathrm{M}$, lysosome inhibitor) nor

3-methyladenine ( $2 \mathrm{mM}$, autophagy inhibitor) blocked Pi-induced reduction of HDAC1. (b) Ubiquitination of HDAC1 was enhanced by Pi treatment for 3 days (fourth versus 5th lane). Twenty-four hours after transfection with Flag-HDAC1 and HA-Ub, A10 cells were treated with Pi. MG132 was added $4 \mathrm{~h}$ before collecting. The cell lysates were immunoprecipitated with anti-HA antibody and immunoblotted with anti-HDAC1 antibody. Western blots (4-14) from four to eight independent sets were analysed. (c) Tandem ubiquitin-binding entities (TUBEs) assay to check K48 ubiquitination of HDAC1 in response to Pi. Pi-treated RVSMCs were subjected to GST pull-down assay with either GST only or GST-TUBE and then HDAC1 was detected with immunoblot. HDAC1-bound multiple ubiquitin conjugation was detected. A10 cells were treated with Pi for 3 days. (d) Von Kossa staining showed that Pi-induced RVSMC calcification was blunted by either MG132 $(10 \mu \mathrm{M})$ or lactacystin $(10 \mu \mathrm{M})$. Scale bar, $100 \mu \mathrm{m}$. (e) Quantification results ( $n=9$ from three sets). (f) Administration of MG132 to mice blunted VD $\mathrm{D}_{3}$-induced VC. MG132 (2.5 $\mathrm{mg} \mathrm{kg}^{-1}$ per day, intraperitoneally) was administered for 9 days, whereas VD was treated for the first 3 days. Scale bar, $250 \mu \mathrm{m}$ (low power); $50 \mu \mathrm{m}$ (high power). (g) Quantification of calcium deposition in proximal aorta (17-22 aortic samples from four to six independent experimental sets). (h) TUBE assay revealed that HDAC1 ubiquitination is dependent on K74. Treatment of A10 cells with Pi for 3 days failed to induce ubiquitination of HDAC1 K74R, whereas it successfully induced ubiquitination of HDAC1 K89R. (i) Decoy peptide spanning HDAC1 K74 attenuated Pi-induced reduction of HDAC1 protein amount (eight samples from two sets). (j) K74 peptide attenuated Pi-induced calcium deposition. Von Kossa staining was performed. Scale bar, $100 \mu \mathrm{m}$. ${ }^{\star} P<0.05,{ }^{\star \star} P<0.01$, NS. not significant. Numerals in bar graphs are the numbers of samples.
} 
The molecular mechanism of VC is somewhat similar to that of osteogenic differentiation. The role of MDM2 in osteoblast differentiation has been reported; bone formation fails in mice lacking MDM2 in osteoblast progenitor cells but can be successfully rescued by $\mathrm{p} 53$ inactivation ${ }^{39}$. We investigated the possible involvement of MDM2-mediated regulation of p53 in VC. $\mathrm{VD}_{3}$ administration reduced $\mathrm{p} 53$ protein amounts in mouse aorta (Supplementary Fig. 7b). In RVSMCs, Pi also reduced p53; however, interestingly, MDM2 siRNA failed to block the Piinduced reduction of p53 (Supplementary Fig. 7c). We further checked whether reduction of p53 affects VC. Reduction of p53 with siRNA (Supplementary Fig. 7d) did not alter the Pi-induced VC (Supplementary Fig. 7e). Likewise, pifithrin- $\alpha$, a p53 inhibitor ${ }^{40}$, did not affect the VC (Supplementary Fig. 7f). a

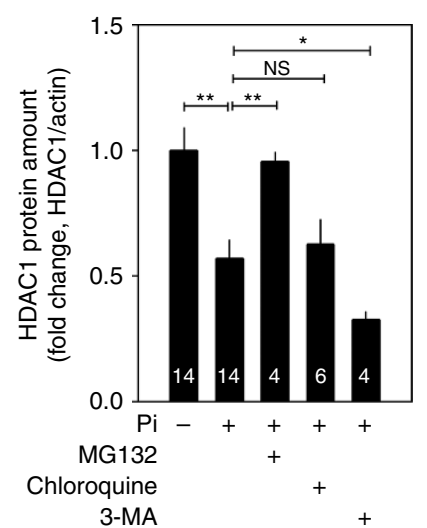

RVSMCs

d
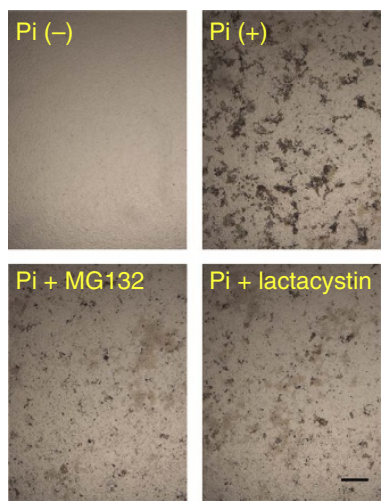

RVSMCs b
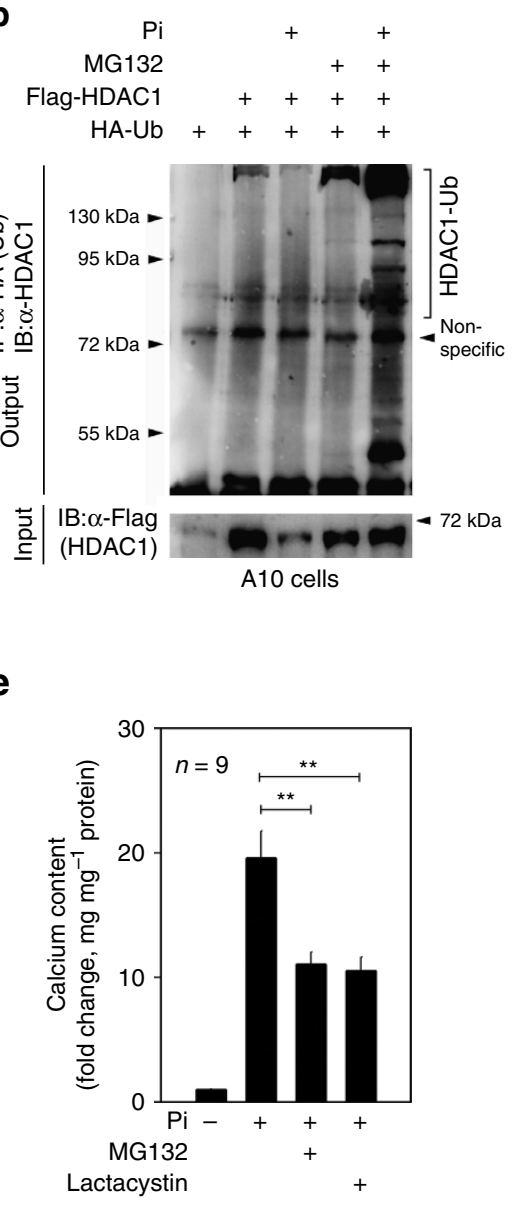

RVSMCs

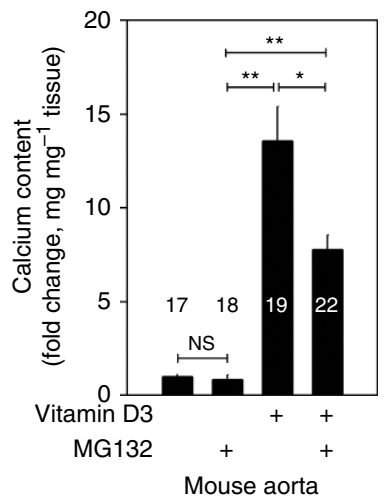

h

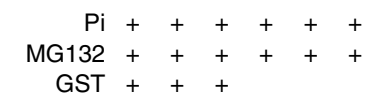
GST-TUBE Flag-HDAC1 WT Flag-HDAC1 K89R Flag-HDAC1 K74R

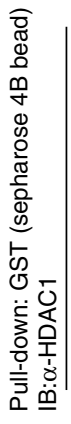
훈 A10 cells
C

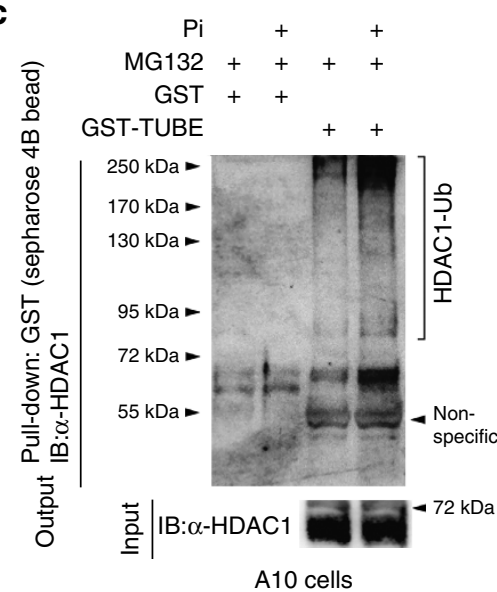

f

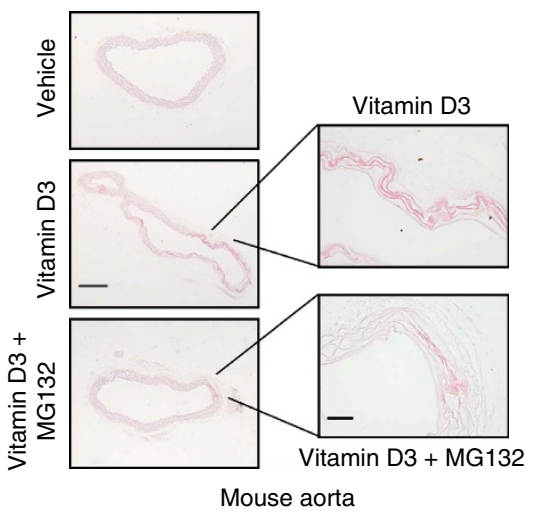

i

j

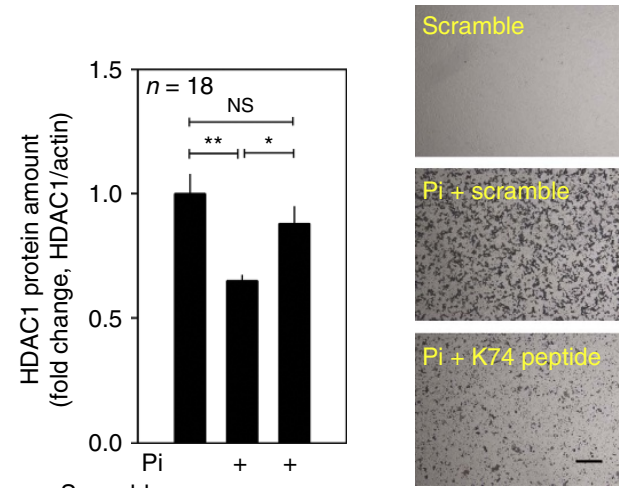

Scramble ++ RVSMCs K74 peptide

\section{RVSMCs}


a

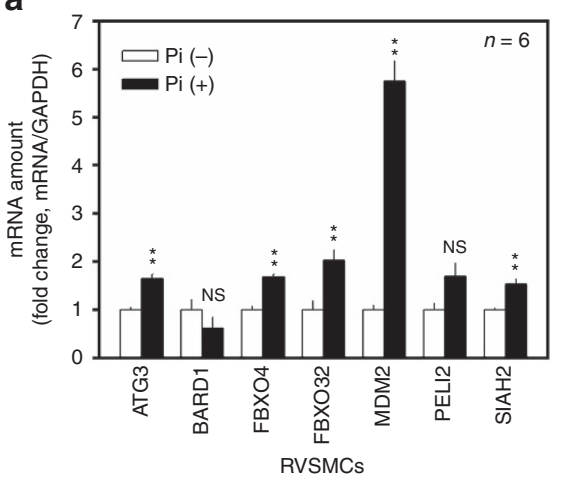

b

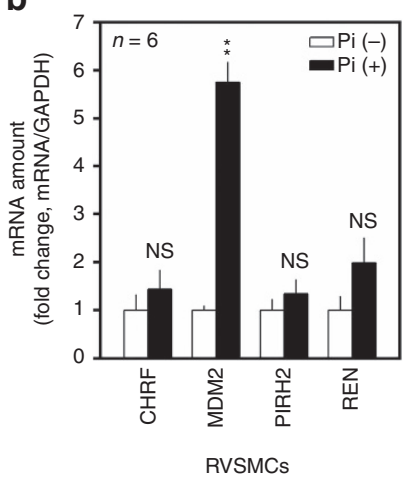

C

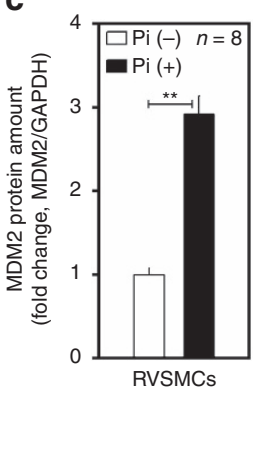

d

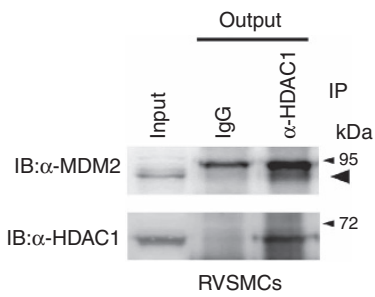

e

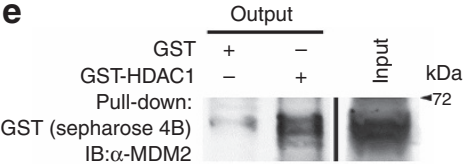

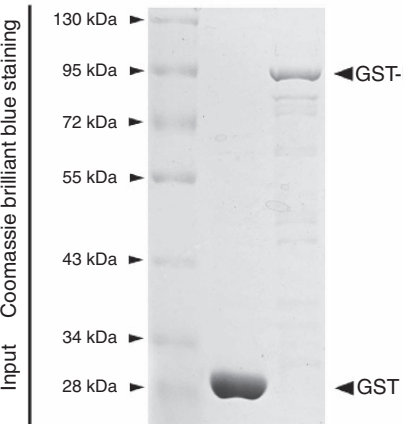

g

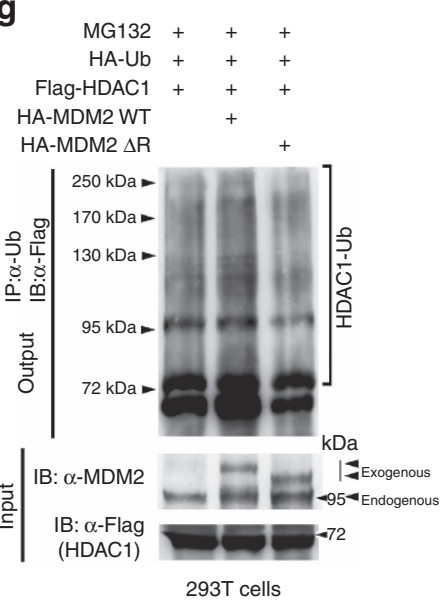

h
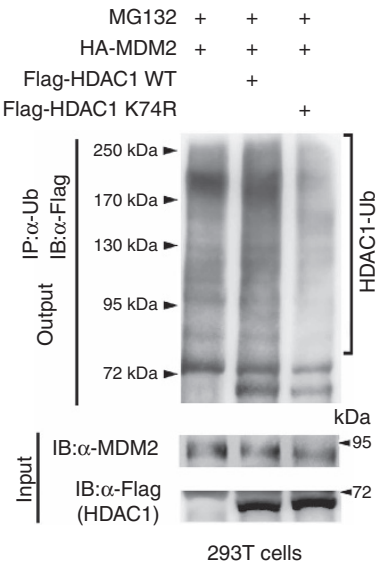

Figure 5 | MDM2 E3 ligase induces degradation of HDAC1. (a) Pi-induced changes in the mRNA level of seven candidate genes according to cDNA microarray analysis ( $n=6$ from two sets). Each sample was measured in duplicate. (b) Pi-induced changes in the mRNA level of four candidate genes on the basis of reports in the literature of HDAC1-specific E3 ligases ( $n=6$ from two sets). Note that MDM2 was listed among both the cDNA microarray-based candidates (a) and the literature-based ones (b). (c) Pi significantly increased MDM2 protein expression ( $n=8$ from two sets).

(d) Immunoprecipitation analysis showed that endogenous HDAC1 physically associated with endogenous MDM2 in RVSMCs. (e) GST pull-down assay to show the direct interaction between MDM2 and HDAC1. Both GST-HDAC1 and His-MDM2 proteins were generated from E. coli and then utilized for GST pull-down assay. MDM2-His was recruited by Sepharose 4B-bound GST-HDAC1. (f) Adenoviral infection of MDM2 induced dose-dependent reduction of HDAC1 protein in RVSMCs. Cells were treated with an equal amount of Ad-HDAC1 (20 MOI) in each case. (g) Transfection of wild-type MDM2 to 2937 cells enhanced the ubiquitination of HDAC1 (second lane). However, transfection of MDM2 $\triangle R$ that lacked the RING domain for E3 ligase activity failed to do so (third lane). Flag-HDAC1 and HA-Ub with either HA-MDM2 or HA-MDM2 2 R were transfected and maintained for 2 days. Cells were treated with MG132 $4 \mathrm{~h}$ before collecting. The cell lysates were immunoprecipitated with Ub and immunoblotted with HDAC1. (h) MDM2-induced HDAC1 ubiquitination was attenuated in HDAC1 K74R. Ub assay was performed. ${ }^{\star \star} P<0.01$, NS, not significant.

These results suggest that MDM2 is not involved in Pi-induced p53 reduction, and that the reduction of p53 does not participate in the $\mathrm{VC}$ at least in our experimental model.

MDM2 causes VC in vivo models. We next studied the function of MDM2 in vivo models. In $\mathrm{VD}_{3}$-administered mouse aorta, the transcript level of MDM2 was significantly increased (Fig. 7a). Histological analysis with quantification also showed that MDM2 was increased in the aorta of $\mathrm{VD}_{3}$-administered mice (Supplementary Fig. 8a,b). MDM2 expression was also increased in both ApoE KO mice fed a diet high in cholesterol and calcium (Fig. 7b) and ApoE $\mathrm{KO}$ carotid artery ligation mice (Supplementary Fig. 8c,d). The increases in MDM2 expression were further examined in two human calcification models of intimal and medial calcification. Histological analysis showed that MDM2 expression was increased in the intimal calcification model (Fig. 7c; Supplementary Fig. 8e). The increase in MDM2 mRNA level was also observed (Fig. 7d). Because metabolic diseases such as diabetes mellitus or chronic renal failure might cause medial calcification ${ }^{23,26}$, we investigated whether the increase in MDM2 is also associated with medial calcification in human coronary artery. We observed significant MDM2-positive signals near the calcification site (Fig. 7e). Indeed, MDM2 protein expression was increased, whereas HDAC1 was downregulated in calcified human coronary artery (Fig. 7f).

Next, we examined whether blockade of MDM2 activity by RG compound could result in the prevention of $\mathrm{VC}$ in $\mathrm{VD}_{3}$ administered mice model. Intraperitoneal administration of RG to mice restored the $\mathrm{VD}_{3}$-induced reduction of $\mathrm{HDACl}$ in the arch of aorta as determined with immunoblot (Fig. 7g). Immunohistochemistry analysis for HDAC1 also showed that HDAC1 expression was restored by administration of RG (Fig. 7h). Most importantly, intraperitoneal administration of $\mathrm{RG}$ blocked the $\mathrm{VD}_{3}$-induced $\mathrm{VC}$ in mouse, as determined by Alizarin red $S$ staining (Fig. 7i). These results suggest that chemical inhibition of MDM2 may prevent VC. 
a

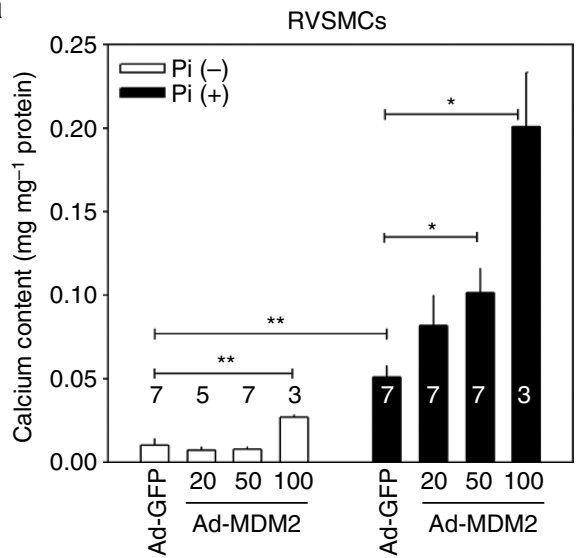

C

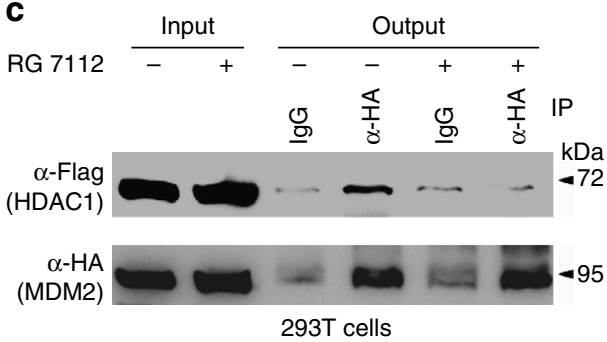

b

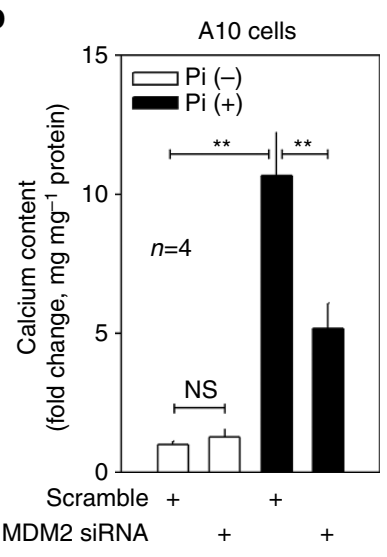

d

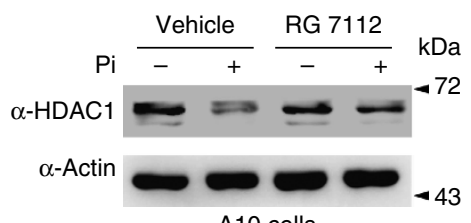

A10 cells e

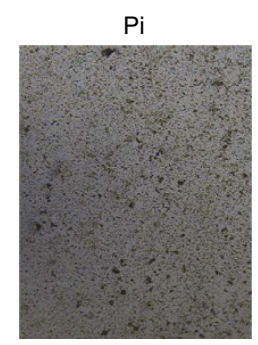

$\mathbf{f}$

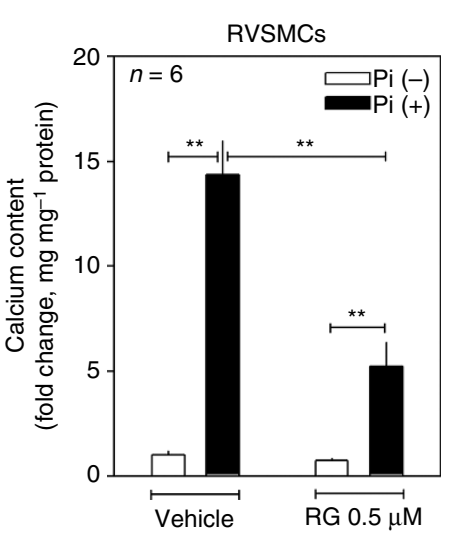

von Kossa staining RVSMCs

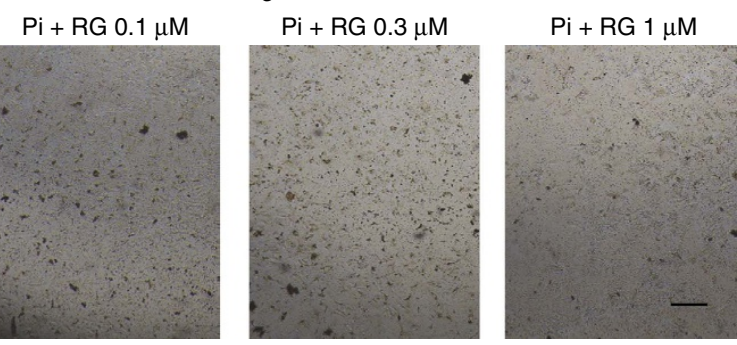

g

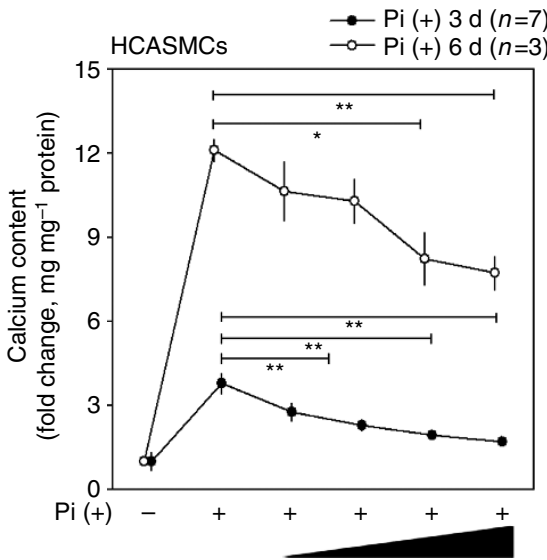

RG 7112

Figure 6 | MDM2 induces VC. (a) Adenoviral infection of MDM2 enhanced Pi-induced VC in a dose-dependent manner. Numbers under the horizontal axis are the MOI of adeno-MDM2 ( $n=3-7$ from one to two experimental sets). (b) MDM2 siRNA blunted Pi-induced VC in A10 cells ( $n=9$ from three sets). Either MDM2 siRNA or scramble was transfected with Lipofectamine RNAiMAX. (c) Immunoprecipitation analysis to show that RG 7112 (RG), an MDM2 inhibitor, interfered with the association of HDAC1 with MDM2. Note the physical interaction between HDAC1 and MDM2 (fourth lane) was attenuated by RG treatment (sixth lane). HA-MDM2 and Flag-HDAC1 were transfected and either RG $(2.5 \mu \mathrm{M})$ or vehicle was treated for $24 \mathrm{~h}$ in $293 \mathrm{~T}$ cells. (d) RG $(0.1 \mu \mathrm{M})$ blocked the Pi-induced reduction of HDAC1 protein amount in A10 cells. (e) RG attenuated Pi-induced VC in RVSMCs in a dose-dependent manner. Pi-containing media with either RG or vehicle were replaced every 2 days for 6 days and von Kossa staining was performed. Scale bar, $100 \mu \mathrm{m}$. (f) Quantification results to show the inhibitory effect of RG on Pi-induced VC. RG $(0.5 \mu \mathrm{M})$ significantly reduced the calcium deposition in RVSMCs ( $n=6$ from two sets). (g) Dose-dependent attenuation of calcium deposition by RG compound (0.1-3 $\mu$ M) in human coronary artery smooth muscle cells (HCASMCs). Pi was treated for 3 days (filled circle, $n=7$ from two sets) or 6 days (open circle, $n=3$ from one set). ${ }^{\star} P<0.05$, ${ }^{\star \star} P<0.01$. 


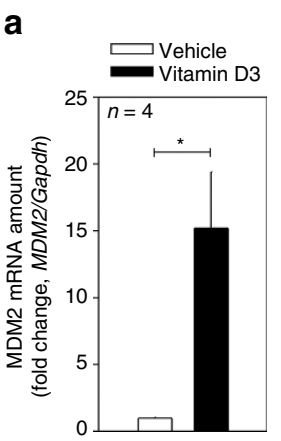

f

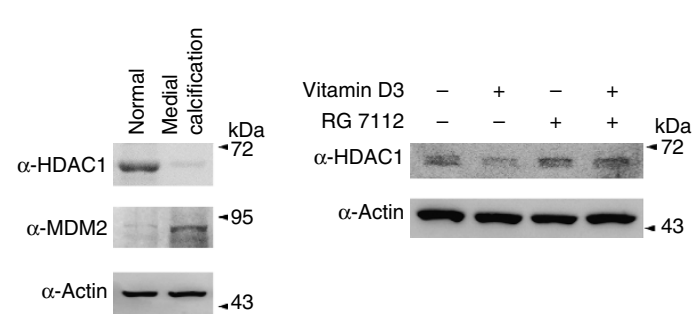

b

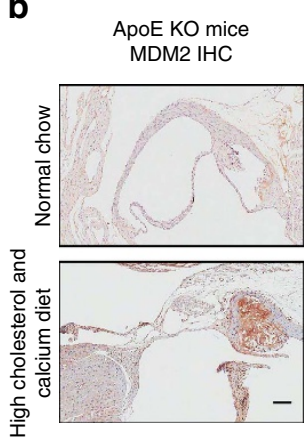

g
C

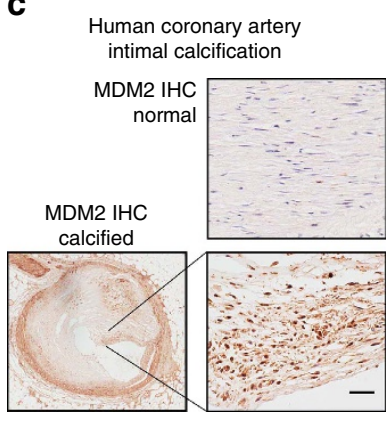

d

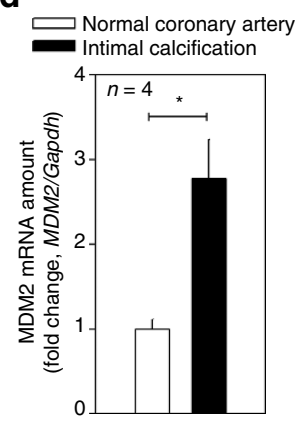

h

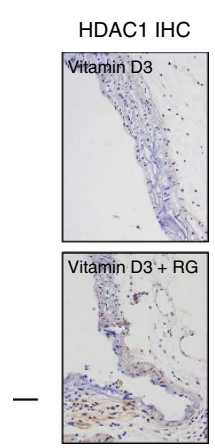

i

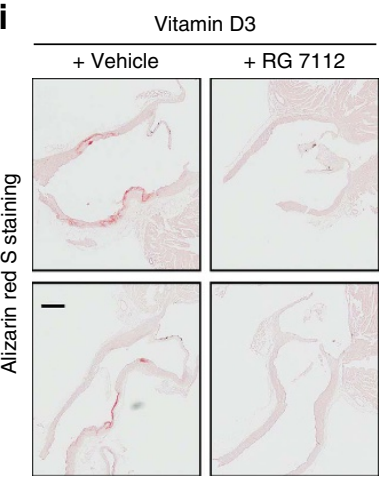

e

Human coronary artery medial calcification

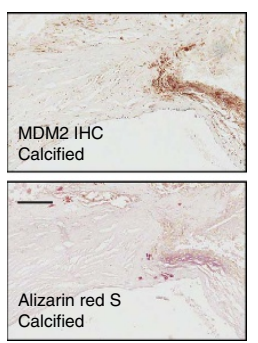

j

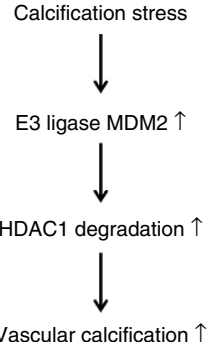

Figure 7 | MDM2 is upregulated in VC models and inhibition of MDM2 activity reduces VC in mice. (a) MDM2 mRNA level was upregulated in the aorta of $\mathrm{VD}_{3}$-administered mice (four mice from two sets). Each sample was measured in duplicate. (b) Immunohistochemical analysis showing MDM2 expression in ApoE mice fed high cholesterol and calcium as explained in Fig. 3f. Scale bar, $100 \mu \mathrm{m}$. (c) Immunohistochemical analysis showing that MDM2 expression is increased in the atherosclerosis-associated intimal calcification model of human coronary artery. The adjacent section slide was used with Fig. 3h. Scale bar, $50 \mu \mathrm{m}$. (d) Quantitative real-time RT-PCR results show that MDM2 mRNA level is significantly increased in the intimal calcification model of human coronary artery (four samples in duplicate). (e) MDM2 expression was increased at the site of calcification in human coronary artery with medial calcification in association with diabetes. Scale bar, $200 \mu \mathrm{m}$. (f) HDAC1 was downregulated, whereas MDM2 was upregulated, in human coronary artery sample with medial calcification. (g) Administration of RG (intraperitoneally, $50 \mathrm{mg} \mathrm{kg}^{-1}$ per day, 9 days) prevented $\mathrm{VD}_{3}$-induced reduction of $\mathrm{HDAC1}$ protein amount in mouse aorta. (h) Immunohistochemical analysis showing HDAC1 expression in $\mathrm{VD}_{3}$-treated mice. Note that nuclear expression of HDAC1 was restored by administration of RG. Scale bar, $50 \mu \mathrm{m}$. (i) Intraperitoneal administration of RG prevented VD-induced VC in the ascending aorta. Scale bar, $250 \mu \mathrm{m}$. (j) Diagram of MDM2/HDAC1 signal cascade in VC. ${ }^{\star} P<0.05$.

\section{Discussion}

Here we elucidate the previously unknown MDM2/HDAC1 pathway in the development of $\mathrm{VC}$ (Fig. 7j). In this pathway, under conditions that lead to calcification, the expression of MDM2 is induced, and MDM2 acts as an E3 ligase to ubiquitinate $\mathrm{HDAC1}$. The reduction of $\mathrm{HDAC1}$ contributes to the potentiation of $\mathrm{VC}$, which is somewhat coincident with our result that HDACi exaggerates VC. These findings show not only an HDAC-related regulation mechanism but also novel MDM2-mediated proteasomal degradation in VC. This observation provides clues for potential therapeutic application of the MDM2/HDAC1 signal cascade, either by utilizing decoy peptide against HDAC1 degradation or by MDM2 inhibitor.

These findings are of major significance. (1) In this report, we postulate that HDACi might have potential adverse effects of VC. (2) We first demonstrated that ubiquitination mediates VC; blockade of proteasomal degradation by diverse inhibitors of ubiquitination attenuated $\mathrm{VC}$ in vitro and in vivo models. (3) We show that MDM2/HDAC1 may represent an attractive target for drug development against $\mathrm{VC}$ by demonstrating the role of MDM2/HDAC1 signalling cascade in the cardiovascular pathophysiology. Indeed, we demonstrated that blockades of either HDAC1 degradation or MDM2 activity can attenuate VC. Since MDM2 inhibitors such as nutlin-3 or RG 7112 are now being under extensive investigation for the treatment of cancer by recent research works, application of those drugs for the treatment of VC may be anticipated.

To date, only one report has shown the possible involvement of ubiquitination in VC; Nedd4 E3 ubiquitin ligase was shown to negatively regulate $\mathrm{Pi}$-induced $\mathrm{VC}$ by ubiquitination of Smad1 in cellular models in vitro ${ }^{41}$. That report contradicts our present work concerning whether ubiquitination may enhance VC; those authors observed the development of VC by specific inhibition of Nedd4. Utilizing in vivo and in vitro models in the present study, however, we found that overall inhibition of ubiquitination either in RVSMCs or in mice prevents VC, which is mediated at least in part by the MDM2-mediated degradation of HDAC1.

MDM2 is known to induce ubiquitination of several target proteins, with the most well-known target of MDM2 E3 ligase being p53 (refs 36,42). Upregulation of MDM2 and repression of p53 activity is highly associated with cancer development ${ }^{43}$. Indeed, MDM2 is clinically utilized as a marker of certain types of soft tissue cancer such as liposarcoma ${ }^{44}$. Thus, MDM2 inhibitors such as RG or nutlin-3 are in phase I clinical trials ${ }^{38}$. In boneselective MDM2 KO mice, the improperly increased p53 activity causes an increase in p21 expression, which results in osteogenic failure in embryo. Thus, during the bone development, MDM2mediated inhibition of p53 activity and the following decrease in p21 transcription are indispensable for the proper expression of Runx 2 in osteoblasts and skeletal development ${ }^{39}$. In contrast, however, in the present study, we observed that p53 was 
downregulated by triggers of calcification in an MDM2indepenent manner, and that the reduction of p53 activity did not cause VC. Thus, in contrast with the p53-dependent pathway in bone formation in embryo, our results strongly suggest that an increase in MDM2 and subsequent reduction of HDAC1 contributes to VC.

It should be noted that the VC models in our experiments, such as VC induced by Pi treatment or high-dose vitamin D3 administration, may not be clinically relevant to human VC. Likewise, the mechanism shown by these models may differ in human VC. The simple phenotypic coincidence of downregulation of $\mathrm{HDAC} 1$ and upregulation of MDM2 in human coronary artery VC samples may not implicate this MDM2/HDAC1 pathway in human VC. Further studies using a large number of human VC models or more clinically relevant animal models are needed to adequately address this issue. Nonetheless, our report does suggest a plausible mechanism of MDM2/HDAC1 in VC.

As transcriptional regulators, $\mathrm{HDACi}$ are potential therapeutics in cancer and neurological disorders. $\mathrm{HDACi}$ also promote osteoblastic maturation ${ }^{45}$. Among the HDACs in osteogenesis process, reduction of HDAC1 in osteoblast induces bone formation by the transcriptional activation of Runx2 (ref. 46), a master regulator of osteogenesis ${ }^{47}$. Although it has not been clearly demonstrated which HDAC is involved, HDACi induce calcification in VSMCs by inducing Runx2 and alkaline phosphatase $^{19}$. In the present study, reduction of HDAC1 and inhibition of HDAC activity induced ectopic calcification in the vasculature. It should be noted that HDACi are being currently used in anticancer therapeutics. In addition, possible clinical applications of HDACi in diverse heart diseases, such as cardiac hypertrophy $16,17,48$, fibrosis ${ }^{11,12}$, arrhythmia ${ }^{13}$ and myocardial infarction 14,15 , and neurologic diseases such as epilepsy ${ }^{49}$, are being highlighted by many research groups including ours. Considering our results, however, the likeliness of increase of VC by HDACi should be noted as a potential adverse effect for future drug development.

\section{Methods}

Reagents. Antibodies against anti-HDAC2 (1:1,000, ab12169), HDAC3 (1:1,000, ab16047), HDAC8 (1:1,000, ab137474), FBXO4 (1:1,000, ab83318) and MDM2 (1:1,000, ab3110) were from Abcam (Abcam, Cambridge, UK); Flag (1:1,000, F7425 and F1804) and actin (1:1,000, A2066) were from Sigma (Sigma-Aldrich, St Louis, MO, USA); HDAC1 (1:1,000, sc7872 and 05-100) was from either Santa Cruz Biotechnology (Santa Cruz Biotechnology, Santa Cruz, CA, USA) or Millipore (EMD Millipore, Billerica, MA, USA); ATG3 (1:1,000, sc-70139), SIAH2 (1:1,000, sc-5507), Ub (1:1,000, sc8017), and Gapdh (1:1,000, sc16574) were from Santa Cruz Biotechnology; Ub $(1: 1,000,3936)$ was from Cell Signaling (Cell Signaling Technology, Denver, MA, USA); FBXO32 (1:1,000, AP2041) was from ECM Biosciences (ECM Biosciences, Versailles, KY, USA); and HA (1:1,000, 11583816001) was from Roche Applied Science (Roche Applied Science, Indianapolis, IL, USA). Anti-mouse (1:5,000, 7076S) or anti-rabbit $(1: 5,000,7074 \mathrm{~S})$ IgG peroxidase-conjugated secondary antibodies were purchased from Sigma (Sigma-Aldrich).

Cholecalciferol (vitamin $\mathrm{D}_{3}$ ), TSA, apicidin, phosphonoformic acid, pifithrin- $\alpha$ and 2,2,2-tribromoethanol were purchased from Sigma (Sigma-Aldrich). MG132 was purchased from Calbiochem (EMD Millipore). Chloroquine, 3-methyladenine, lactacystin and epoxomicin were purchased from Sigma (Sigma-Aldrich). $N$-[N-(N-Acetyl-L-leucyl)-L-leucyl]-L-norleucine was purchased from Santa Cruz Biotechnology. HDAC1 siRNA, HDAC2 siRNA, MDM2 siRNA and scramble were purchased from Dharmacon (Thermo Fisher Scientific, Waltham, MA, USA). p53 siRNA was purchased from Bioneer (Daejeon, Korea). Decoy peptide was synthesized by Peptron (Daejeon, Korea). RG 7112 compound was purchased from ApexBio Technology (Apexbio Technology LLC, Houston, TX, USA).

Plasmids. pBJ5.1-Flag-HDAC1 was kindly gifted from Professor Jonathan A. Epstein (University of Pennsylvania, Philadelphia, PA, USA). $p C M V-M D M 2$ and pcDNA3-HA-Ub were kindly provided by Dr Ki Sun Kwon (Korea Research Institute of Bioscience \& Biotechnology, Daejeon, Korea). pcDNA6-3xHA-MDM2-Myc, pGEX-HDAC1 and pET28-MDM2 were generated by PCR-based subcloning (CosmoGeneTech, Seoul, Korea). RING finger domain-deleted pcDNA6-3xHAMDM2-Myc (MDM2AR), pBJ5.1-Flag-HDAC1 K74R and pBJ5.1-Flag-HDAC1
$K 89 R$ were constructed by site-directed mutagenesis (CosmoGeneTech). All plasmids were checked before the use by direct sequencing.

Cell cultures. RVSMCs were isolated from rat thoracic aorta of 6-7-week-old Sprague-Dawley male rats. The aorta was washed using sterilized ice-cold PBS and incubated in $1 \mathrm{ml} 0.2 \%$ collagenase I solution in Ham's F12 medium at $37^{\circ} \mathrm{C}$ for $30 \mathrm{~min}$. The aortas were opened longitudinally and the intima scraped on luminal surface. Tissue samples were minced into small pieces in dissection medium with Ham's F12 media containing $300 \mathrm{U} \mathrm{ml}^{-1}$ penicillin and $300 \mathrm{U} \mathrm{ml}^{-1}$ streptomycin, and incubated the dissected tissues in $0.2 \%$ collagenase I solution at $37^{\circ} \mathrm{C}$ for 30 min with shaking. The dissected tissues were attached to dish and cultured $10 \%$ fetal bovine serum (FBS) in DMEM with antibiotics at $37^{\circ} \mathrm{C}$ in a humidified atmosphere with $5 \% \mathrm{CO}_{2}$. RVSMCs were used at passages 2-6. A10 cells were purchased from American Type Culture Collection (CRL-1476, Manassas, VA, USA) and have been used as models of RVSMCs ${ }^{50}$. A10 cells were derived from embryonic rat aorta ${ }^{51}$ and were maintained in $10 \%$ FBS in DMEM with antibiotics Human embryonic kidney 293T cells were obtained from the Seoul Korean Cell Line Bank (21573, Seoul, Korea) and were maintained in 10\% FBS in DMEM with antibiotics. HCASMCs were purchased from Gibco (Invitrogen, C-017-5C, Carlsbad, CA, USA) and were grown in Medium 231 with smooth muscle growth supplement.

Adenoviral GFP, HDAC1 and MDM2. HDAC1 (096643A) and MDM2 (106880A) adenovirus were purchased from Applied Biological Materials (Richmond, BC, Canada). Titre of adenoviruses was determined by the use of the Adeno-X Rapid Titer kit (Clontech Laboratories, Mountain View, CA, USA). The expression of protein was examined by the infection of adenoviral GFP.

cDNA microarray. To investigate the alteration of the expression of E3 ligase in response to calcification stresses, calcification was induced in RVSMCs treated with inorganic phosphate $(\mathrm{Pi})$ and the mRNA preparation was subjected to cDNA microarray analysis (Rat GE $4 \times 44 \mathrm{k}$ v3 microarray chip, G2519F, Agilent, Santa Clara, CA, USA). To reduce the variation between sample preparations, two independent sets of experiments were performed and analysed (Genomictree, Daejeon, Korea). Gene expression data have been deposited in the GEO database under accession code GSE74755.

Induction of VC in vitro and ex vivo. For the induction of VC in RVSMCs, the cells cultured in growth medium were switched to calcification medium containing $2 \mathrm{mM} \mathrm{Pi}(\mathrm{pH} 7.4$ ) for up to 3 or 6 days. The medium was changed every 2 days. The first day of culture in the calcification medium was defined as day 0 and calcium deposition was determined after the cells were washed twice with $1 \times$ PBS at day 6 .

VC was also induced by treatment of RVSMCs with OM. OM was generated by adding $100 \mathrm{nM}$ dexamethasone, $1 \mu \mathrm{M}$ insulin, $50 \mu \mathrm{g} \mathrm{ml}^{-1}$ ascorbic acid and $10 \mathrm{mM} \beta$-glycerophosphate with $8 \mathrm{mM} \mathrm{CaCl}_{2}$ to culture media. VC was induced for 3-21 days.

For ex vivo experiments, the 6-week-old C57BL/6 male mice were killed under anaesthesia with 2,2,2-tribromoethanol ( $300 \mathrm{mg} \mathrm{kg}^{-1}$, intraperitoneally). The heart and thoracic aortas were dissected down to the renal arteries and removed in $1 \times$ PBS including antibiotics. The heart and aortic root were separated from the more distal aorta ${ }^{52}$. The separated aortas were plated on cell culture plates $(60 \mathrm{~mm})$ and cultured in DMEM with 10\% FBS and penicillin-streptomycin solution (growth medium). The aortas were then switched to a growth medium containing $2 \mathrm{mM} \mathrm{Pi}$ ( $\mathrm{pH}$ 7.4) for up to 6 days. The medium was changed every 2 days. The first day of culture in the calcification medium was defined as day 0 . The aortas were washed twice with $1 \times$ PBS and calcium deposition was determined.

Either K74 decoy peptide or scramble was treated to RVSMCs at the concentration of $100 \mathrm{nM}$ for 6 days. The peptide was replenished every 2 days when the Pi-containing culture media was changed.

Quantification of calcium deposition. Cells and tissues were decalcification with $0.6 \mathrm{~N} \mathrm{HCl}$ at $4{ }^{\circ} \mathrm{C}$ for $24 \mathrm{~h}$. The calcium content of the $\mathrm{HCl}$ supernatants was determined colorimetrically using Calcium assay kit (QuantiChromTM Calcium Assay Kit, BioAssay Systems, Hayward, WI, USA) according to the manufacturer's recommendations. Briefly, $5 \mu \mathrm{l}$ of the samples was transferred to a 96 -well plate. Working reagent $(200 \mu \mathrm{l})$ was added and absorbance was then measured at $570 \mathrm{~nm}$ using a microplate ELISA reader (BioTek Instruments, Winooski, VT, USA). After decalcification, cells were washed three times with PBS and solubilized with $0.1 \mathrm{~N}$ $\mathrm{NaOH} / 0.1 \%$ SDS. The protein content was measured with a BCA Protein Assay kit (Thermo Scientific Pierce, Rockford, IL, USA). The calcium content of RVSMCs or A10 cells was then normalized to the protein content, whereas that of the tissues was normalized to tissue dry weight.

For serum calcium measurement, blood samples were obtained from mice and kept on ice overnight and used for assay after centrifugation at 10,000 r.p.m. for $30 \mathrm{~min}$. Serum calcium was assayed using the QuantiChrom calcium assay kit (BioAssay Systems). 
Determination of calcium deposition by calcium staining. Tissues to be stained for calcifications were collected and stored in $70 \%$ ethanol. Alizarin red staining was used to stain soft tissue calcifications. Each individual tissue sample was placed in $10 \mathrm{ml}$ of alizarin working solution that contained $0.8 \%$ Alizarin red S in $0.5 \%$ $\mathrm{KOH}$ and was rotated for $24 \mathrm{~h}$. The tissues were then removed from the Alizarin working solution, replaced in $10 \mathrm{ml}$ of $0.05 \% \mathrm{KOH}$ and rotated for another $24 \mathrm{~h}$ to remove any unbound stain from the tissues. A photograph was then taken to record the data.

For detection of mineralization of RVSMCs, von Kossa staining was performed ${ }^{53}$. In brief, cells were fixed with $10 \%$ formalin for $30 \mathrm{~min}$ at room temperature, washed with $\mathrm{dH}_{2} \mathrm{O}$ three times and then incubated with $5 \%$ silver nitrate for $30 \mathrm{~min}$ at room temperature. The cells were exposed to ultraviolet light for $2 \mathrm{~h}$ or overnight until colour development was complete. The silver nitrate solution was removed and the cells were washed with double distilled $\mathrm{H}_{2} \mathrm{O}$ and photographed by microscopy (Carl Zeiss, Jena, Germany).

Quantitative real-time PCR. Total RNA from either cells or tissues was extracted using TRIzol Reagent (Invitrogen, Grand Island, NY, USA). The cDNA synthesis was generated using iScript cDNA Synthesis Kit (Bio-Rad Laboratories, Hercules, CA, USA) and analysed by real-time qPCR using a QuantiTech SYBR Green RT-PCR Master Mix (Qiagen, Valencia, CA, USA) and a Rotor gene Q (Qiagen, Hilden, Germany). All data were normalized to GAPDH. To rule out possible genomic DNA contamination, primers were designed to include an intervening intron. Amplimers were sequenced for confirmation. Primers for real-time qRT-PCR were as follows: RUNX2: $5^{\prime}$-CGCCTCACAAACAACCACAGAACC- ${ }^{\prime}$, 5'-TGCTGCTGCTGTTGCTGCTGCTGC-3'; HDAC1: 5' - AGGGCACCAAGAG GAAAGTCTGTT-3' $5^{\prime}$ - TTCAGACTTCTTCGCATGGTGCAG-3'; HDAC2: 5'-GCCAACCCCGCTCTGCGATC-3', $5^{\prime}$-GCCGCCTCCTTGACTGTACGC-3'; HDAC3: 5'-CAAGACCGTGGCGTATTTCTACGA-3', 5'-GCCCAGTTGATGGC AATATCACAG-3'; HDAC4: 5'-GCATCCCTGTGTCATTTGGC-3', 5'-CAGCG AGCTGTCCAGTTTCT-3'; HDAC5: 5'-TCCCGTCCGTCTGTCTGTTA-3', 5'-GACATGCCATCCGACTCGTT-3'; HDAC6: 5'-CGAGTTCTTGCAGGCAC CTA-3', 5'-ATGCTCATAGCGGTGGATGG-3'; HDAC7: 5'-TGCTGGAGAAAG AAGAGATGATT-3' ${ }^{\prime} 5^{\prime}$-GCAAGCCTGCTAGGAAGAGAT-3'; HDAC8: $5^{\prime}$-AGT TGGACGAGGGACTAGGG-3', 5' -CTATTGGCGGGTTCCTCTGG-3'; HDAC9: 5'-ACCTACCGACAGTAGCAGCC-3', 5' -TCCGGCCACTCCATCTGATT-3'; Chfr; 5' -AGGATTCCCGCATCGCCCCT-3', $5^{\prime}$-ACAGTTGCGGCCCCAGT AGC-3'; MDM2: 5'-GCGAGCGGAGACGGACACAC-3' 5 $^{\prime}$-GGGCTCTGTGGC GCTTCCTC-3'; REN: $5^{\prime}$-TGCGACAACCCAGGAAGGCG-3' $5^{\prime}$-CGGTCGGAC GCTGCCTTCAG-3'; PIRH2: 5'-CCCTTCGCCCCGCAATCTGG-3' 5' $^{\prime}$-CCAGC TTGGAAGCCACGCCT-3'; ATG3: 5'-ATTGCGACAGTCTCTCCGTG-3', 5'-ACACCGCTTGTAGCATGGAA-3'; BARD1: $5^{\prime}$-GGTGTTACTGTCCCACG GAG-3', 5'-CTTGGGCTTTCTGCTGAGGA-3'; FBXO4: 5'-CTTGAAAGCCAG CCGTCCTA-3 ${ }^{\prime}, 5^{\prime}$-CCTCGCTCTGTAGGCTGAAC- ${ }^{\prime}$; FBXO32: $5^{\prime}$-CACTTCTC AGAGCGGCAGAT- $3^{\prime}, 5^{\prime}$-AGCAGCTCTCTGGGTTGTTG-3'; SIAH2: $5^{\prime}$-TAAC CAATGCCGCCAGAAGT-3', 5'-GCATCATCACCCAGTCCACA-3'; PELI1: $5^{\prime}$-TCTCAAGGCTCCTGACCAGT- ${ }^{\prime}$ and $5^{\prime}$-TGTTGCTTATTGCCTTGGCG- ${ }^{\prime}$.

Immunoprecipitation and western blot analysis. Cells and tissues were collected with lysis buffer ((50 mM Tris (pH 8.0), $150 \mathrm{mM} \mathrm{NaCl}, 1 \mathrm{mM}$ EDTA, $1 \% \mathrm{NP}-40$, $1 \mathrm{mM}$ DTT, $1 \mathrm{mM}$ phenylmethylsulfonyl fluoride, $1 \mathrm{mM} \mathrm{Na}_{3} \mathrm{VO}_{4}$ and $1 \mu \mathrm{g} \mathrm{ml}{ }^{-1}$ each of leupeptin, pepstatin and aprotinin). One milligram of proteins was then immunoprecipitated overnight at $4^{\circ} \mathrm{C}$ with the indicated antibody. After extensive washing with lysis buffer, the immunocomplexes were analysed by western blotting assay. The lysates were separated by SDS-polyacrylamide gel electrophoresis and transferred overnight at $100 \mathrm{~mA}$ on to a polyvinylidene difluoride membrane (Millipore, Bedford, MA, USA) and blocked with 5\% skim milk in $1 \times$ TBST. Membranes were incubated with the specific primary antibodies overnight at $4^{\circ} \mathrm{C}$. After three washes in $1 \times$ TBST, membranes were incubated with horseradish peroxidase-linked secondary antibodies for $1 \mathrm{~h}$ at room temperature. Membranes were again washed three times in $1 \times$ TBST, and protein bands were visualized by enhanced chemiluminescence using a Fuji LAS-3000 system (Fujifilm Life Science, Tokyo, Japan).

GST pull-down assay. To investigate the direct interaction between MDM2 and HDAC1, an in vitro binding (GST pull-down) assay was performed. Both recombinant GST-HDAC1 and His-MDM2 proteins were obtained from E. coli transformed with pGEX4T-HDAC1 and PET28-MDM2. Either GST or GST-HDAC1 was immobilized on a Sepharose $4 \mathrm{~B}$ column. After the elution, His-MDM2 was applied to the column. The bound MDM2 was detected with western blot analysis using $\alpha$-MDM2 antibody.

Ubiquitination assay. To detect ubiquitinated $\mathrm{HDAC} 1$ proteins, cells in a $10-\mathrm{cm}$ plate were transiently transfected with $4 \mu \mathrm{g} H A$-ubiquitin expression plasmids together with the indicated plasmid. Six hours before collecting, cells were treated with $25 \mu \mathrm{M}$ of MG132. Samples were then lysed using a 1\% NP-40 lysis buffer ( $50 \mathrm{mM}$ Tris pH 7.5, $150 \mathrm{mM} \mathrm{NaCl}, 5 \mathrm{mM}$ EDTA, $50 \mathrm{mM}$ NaF, $1 \%$ NP- $40,5 \mathrm{mM}$ NEM, $1 \mathrm{mM}$ orthovanadate $\left(\mathrm{Na}_{3} \mathrm{VO}_{4}\right)$ and $1 \mathrm{mM}$ phenylmethylsulfonyl fluoride). The following procedures were identical to the immunoprecipitation assay except for the use of monoclonal anti-ubiquitin antibody.
Tandem ubiquitin-binding entities assay. Commercially available kits (TUBE1 and TUBE2, LifeSensors, Malvern, PA, USA) were utilized according to the manufacturer's protocol with slight modification. For the TUBE assay, A10 cells were treated with Pi and MG132 and the cell lysates were used for the assay. Either GST or GST-TUBE2 was loaded on Sepharose 4B beads and the cell lysates were applied and assayed as in the conventional GST pull-down method. The precipitates were then separated on an SDS-polyacrylamide gel electrophoresis gel and transferred to the polyvinylidene difluoride membrane. The membrane was blocked with $5 \%$ bovine serum albumin and probed with either anti-HDAC1 or anti-Flag antibody.

Induction of VC in mice. Six- to seven-week-old C57BL/6 male mice were used for $\mathrm{VC}$ induction by administration of $\mathrm{VD}_{3}$ as described previously $y^{54} . \mathrm{VD}_{3}(14.575 \mathrm{mg}$, $5 \times 10^{5} \mathrm{IU} \mathrm{kg}^{-1}$ per day) in $70 \mu \mathrm{l}$ of absolute ethanol was mixed with $500 \mu \mathrm{l}$ Cremophor (Alkamuls EL-620, Sigma-Aldrich) for $15 \mathrm{~min}$ at room temperature, and this solution was then mixed with $6.2 \mathrm{ml}$ sterilized water containing $250 \mathrm{mg}$ of dextrose for an additional $15 \mathrm{~min}$ at room temperature. The mice were injected with a dose of $\mathrm{VD}_{3}\left(150 \mu \mathrm{l} 25 \mathrm{~g}^{-1}, 5 \times 10^{5} \mathrm{IU} \mathrm{kg}^{-1}\right.$ per day) subcutaneously for 3 days 55 .

To induction of VC in ApoE KO mice, 10-week-old ApoE KO male mice were fed a high-cholesterol diet for 10 weeks and were then fed a high-cholesterol plus calcium supplement diet for the following 7 weeks. VC was evaluated by Alizarin red S staining in transverse sections of the heart and aorta.

The atherosclerosis model was generated ${ }^{56}$. Subtotal occlusion of the left carotid artery was carried out in male ApoE KO mice ${ }^{57}$. A ventral midline incision was made in the neck. The left carotid artery was exposed and three of four caudal branches of the left carotid artery (left external carotid, internal carotid and occipital artery) were ligated with 7-0 silk suture under a surgical microscope, while the superior thyroid artery was lifted intact. The incision was then closed and the mice were monitored until recovery. After surgery, $A p o E \mathrm{KO}$ mice were fed Paigen's Atherogenic Rodent Diet (D12336; Research Diets, New Brunswick, NJ, USA) for 2 weeks until they were killed.

Administration of MG132 or RG 7112 to mice. MG132 $\left(2.5 \mathrm{mg} \mathrm{kg}^{-1} \text { per day }\right)^{58}$, RG $7112\left(50 \mathrm{mg} \mathrm{kg}^{-1} \text { per day }\right)^{59}$, or appropriate vehicle or scramble was intraperitoneally administered to mice. All of these reagents were administered simultaneously with $\mathrm{VD}_{3}$ for the first 3 days and then the reagent alone at the same dose was administered for the next 6 days until the animals were killed.

Smooth muscle-specific KO of HDAC1 and ApoE KO mice. Floxed HDAC was kindly provided by Professor Eric N. Olson of the University of Texas Southwestern Medical Center ${ }^{60}$. Smooth muscle (SM)22 $\alpha$-cre mice were purchased from Jackson Lab (cat.017491, Bar Harbor, ME, USA). Vascular smooth muscle-specific $H D A C 1 \mathrm{KO}$ mice were generated by breeding the floxed $H D A C 1$ mice with $S M 22 \alpha$-cre mice. Deletion of $H D A C 1$ was confirmed by PCR-based genotyping ${ }^{61}$. The primers were as follows: HDAC1 floxed: $5^{\prime}$-GCCTCTGCTTCC TTAGTGTTGG-3' ${ }^{\prime}, 5^{\prime}$-GAGCAAGGAAAGAGCACAAGCCTG; SM22 $\alpha$ cre: $5^{\prime}$-ATTCTCCCACCGTCAGTACG-3' ${ }^{\prime} 5^{\prime}$-CGTTTTCTGAGCATACCTGGA-3 ${ }^{\prime}$. ApoE KO mice were purchased from Jung Ang Animal (Central Lab, Animal Inc., Seoul, Korea).

Microcomputed tomography. Isolated heart and aorta samples were subjected to microcomputed tomography (Skyscan, Kontich, Belgium ${ }^{62}$. The X-ray source was set at $50 \mathrm{kV}$ and $200 \mu \mathrm{A}$ with a pixel size of $17.09 \mu \mathrm{m}$. The exposure time was $1.2 \mathrm{~s}$. Four-hundred fifty projections were acquired over an angular range of $180^{\circ}$ (angular step of $0.4^{\circ}$ ). The tomographic acquired images were transformed into a sliced volumetric reconstruction by using the Nrecon program (Skyscan) and were analysed using three-dimensional Mimics imaging program (version 14.0, Materialise N.V., Leuven, Belgium).

Human samples. Two different models of both intimal calcification and medial calcification were used. Atherosclerosis-associated VC samples were obtained from autopsied heart patients who died of myocardial infarction, whereas medial calcification samples were obtained from autopsied heart died of diabetes mellitus complications. Age-matched normal coronary artery was used for the control. For the atherosclerosis-associated intimal VC sample, paraffin-embedded left ventricle specimens and left anterior descending coronary artery were provided by the Department of Forensic Medicine, Chonnam National University Medical School. The samples had been obtained from eight individuals: four males with VC and four age-matched males. VC was diagnosed according to microscopic findings.

Human coronary arteries with intimal and medial calcification were obtained from autopsied hearts, and age-matched normal coronary arteries were used for the control. As samples were obtained from autopsied tissues, the written consent was not required. Exemption of subjects' written consent and approval for the use of the samples were obtained from the institutional review board of Chonnam National University Hospital (CNUH-2013-106). 
Histology. Mouse aorta and human coronary artery samples were fixed with $4 \%$ paraformaldehyde and embedded in paraffin. Cross-sections $(5 \mu \mathrm{m})$ were stained with haematoxylin and eosin (H\&E), Alizarin red S and immunohistochemistry with indicated primary antibody.

Quantification of histological images was performed after acquisition of the image by the use of iSolution FL (iMT technology, Canada) software. Two images from each sample were taken from the calcification adjacent area. The number of either HDAC1-positive or MDM2-positive nuclei were counted and divided by the total number of hematoxylin-positive nuclei.

Statistical analysis. Data are represented as means \pm s.e.m. The data were analysed by the use of either the unpaired Student's $t$-test or one-way analysis of variance, which was followed by the Tukey honestly significant difference multiple-comparison post hoc test. When the Levene test for unequal variance was significant, Dunnett T3 was used as a post hoc test. Statistical analysis was performed with PASW Statistics 21 (SPSS, IBM Company, Chicago, IL).

Full blotting images corresponding to the immublottings shown in the main and Supplementary Figs are provided as Supplementary Fig. 9.

\section{References}

1. Frink, R. J., Achor, R. W., Brown, Jr A. L., Kincaid, O. W. \& Brandenburg, R. O. Significance of calcification of the coronary arteries. Am. J. Cardiol. 26, 241-247 (1970)

2. Fitzgerald, P. J., Ports, T. A. \& Yock, P. G. Contribution of localized calcium deposits to dissection after angioplasty. An observational study using intravascular ultrasound. Circulation 86, 64-70 (1992).

3. Giachelli, C. M. Ectopic calcification: gathering hard facts about soft tissue mineralization. Am. J. Pathol. 154, 671-675 (1999).

4. Luo, G. et al. Spontaneous calcification of arteries and cartilage in mice lacking matrix GLA protein. Nature 386, 78-81 (1997).

5. Kuro-o, M. et al. Mutation of the mouse klotho gene leads to a syndrome resembling ageing. Nature 390, 45-51 (1997).

6. Lim, K. et al. Vascular Klotho deficiency potentiates the development of human artery calcification and mediates resistance to fibroblast growth factor 23 . Circulation 125, 2243-2255 (2012).

7. Spicer, S. S., Lewis, S. E., Tashian, R. E. \& Schulte, B. A. Mice carrying a CAR-2 null allele lack carbonic anhydrase II immunohistochemically and show vascular calcification. Am. J. Pathol. 134, 947-954 (1989).

8. Thornell, L., Carlsson, L., Li, Z., Mericskay, M. \& Paulin, D. Null mutation in the desmin gene gives rise to a cardiomyopathy. J. Mol. Cell. Cardiol. 29, 2107-2124 (1997)

9. Bucay, N. et al. osteoprotegerin-deficient mice develop early onset osteoporosis and arterial calcification. Genes Dev. 12, 1260-1268 (1998).

10. Minucci, S. \& Pelicci, P. G. Histone deacetylase inhibitors and the promise of epigenetic (and more) treatments for cancer. Nat. Rev. Cancer 6, 38-51 (2006).

11. Williams, S. M. et al. Class I HDACs regulate angiotensin II-dependent cardiac fibrosis via fibroblasts and circulating fibrocytes. J. Mol. Cell. Cardiol. 67, $112-125$ (2014).

12. Guo, W., Shan, B., Klingsberg, R. C., Qin, X. \& Lasky, J. A. Abrogation of TGF-beta1-induced fibroblast-myofibroblast differentiation by histone deacetylase inhibition. Am. J. Physiol. Lung. Cell. Mol. Physiol. 297, L864-L870 (2009).

13. Risebro, C. A. et al. Epistatic rescue of Nkx2.5 adult cardiac conduction disease phenotypes by prospero-related homeobox protein 1 and HDAC3. Circ. Res. 111, e19-e31 (2012).

14. Lee, T. M., Lin, M. S. \& Chang, N. C. Inhibition of histone deacetylase on ventricular remodeling in infarcted rats. Am. J. Physiol. Heart Circ. Physiol. 293, H968-H977 (2007).

15. Granger, A. et al. Histone deacetylase inhibition reduces myocardial ischemia-reperfusion injury in mice. FASEB J. 22, 3549-3560 (2008).

16. Kee, H. J. et al. Inhibition of histone deacetylation blocks cardiac hypertrophy induced by angiotensin II infusion and aortic banding. Circulation 113, 51-59 (2006).

17. Kong, Y. et al. Suppression of class I and II histone deacetylases blunts pressure-overload cardiac hypertrophy. Circulation 113, 2579-2588 (2006).

18. Gallo, P. et al. Inhibition of class I histone deacetylase with an apicidin derivative prevents cardiac hypertrophy and failure. Cardiovasc. Res. 80, 416-424 (2008).

19. Azechi, T. et al. Trichostatin A, an HDAC class I/II inhibitor, promotes Pi-induced vascular calcification via up-regulation of the expression of alkaline phosphatase. J. Atheroscler. Thromb. 20, 538-547 (2013).

20. Sun, Y. et al. Smooth muscle cell-specific runx2 deficiency inhibits vascular calcification. Circ. Res. 111, 543-552 (2012).

21. Lagger, G. et al. Essential function of histone deacetylase 1 in proliferation control and CDK inhibitor repression. EMBO J. 21, 2672-2681 (2002).

22. Trion, A., Schutte-Bart, C., Bax, W. H., Jukema, J. W. \& van der Laarse, A. Modulation of calcification of vascular smooth muscle cells in culture by calcium antagonists, statins, and their combination. Mol. Cell. Biochem. 308, 25-33 (2008).

23. Giachelli, C. M. et al. Vascular calcification and inorganic phosphate. Am. J. Kidney Dis. 38, S34-S37 (2001).

24. Shao, J. S., Cai, J. \& Towler, D. A. Molecular mechanisms of vascular calcification: lessons learned from the aorta. Arterioscler. Thromb. Vasc. Biol. 26, 1423-1430 (2006).

25. Cho, H. J. et al. Vascular calcifying progenitor cells possess bidirectional differentiation potentials. PLoS Biol. 11, e1001534 (2013).

26. Abedin, M., Tintut, Y. \& Demer, L. L. Vascular calcification: mechanisms and clinical ramifications. Arterioscler. Thromb. Vasc. Biol. 24, 1161-1170 (2004).

27. Ivan, E. et al. Expansive arterial remodeling is associated with increased neointimal macrophage foam cell content: the murine model of macrophagerich carotid artery lesions. Circulation 105, 2686-2691 (2002).

28. Hjerpe, R. et al. Efficient protection and isolation of ubiquitylated proteins using tandem ubiquitin-binding entities. EMBO Rep. 10, 1250-1258 (2009).

29. Hicke, L., Schubert, H. L. \& Hill, C. P. Ubiquitin-binding domains. Nat. Rev. Mol. Cell Biol. 6, 610-621 (2005).

30. Cutrona, G. et al. Effects in live cells of a c-myc anti-gene PNA linked to a nuclear localization signal. Nat. Biotechnol. 18, 300-303 (2000).

31. Oh, Y. M. et al. Chfr is linked to tumour metastasis through the downregulation of HDAC1. Nat. Cell Biol. 11, 295-302 (2009).

32. Gaughan, L., Logan, I. R., Neal, D. E. \& Robson, C. N. Regulation of androgen receptor and histone deacetylase 1 by Mdm2-mediated ubiquitylation. Nucleic Acids Res. 33, 13-26 (2005).

33. Logan, I. R. et al. Human PIRH2 enhances androgen receptor signaling through inhibition of histone deacetylase 1 and is overexpressed in prostate cancer. Mol. Cell. Biol. 26, 6502-6510 (2006).

34. Canettieri, G. et al. Histone deacetylase and Cullin3-REN(KCTD11) ubiquitin ligase interplay regulates Hedgehog signalling through Gli acetylation. Nat. Cell Biol. 12, 132-142 (2010)

35. Deshaies, R. J. \& Joazeiro, C. A. RING domain E3 ubiquitin ligases. Annu. Rev. Biochem. 78, 399-434 (2009).

36. Fang, S., Jensen, J. P., Ludwig, R. L., Vousden, K. H. \& Weissman, A. M. Mdm2 is a RING finger-dependent ubiquitin protein ligase for itself and p53. J. Biol. Chem. 275, 8945-8951 (2000).

37. Vassilev, L. T. et al. In vivo activation of the p53 pathway by small-molecule antagonists of MDM2. Science 303, 844-848 (2004).

38. Tovar, C. et al. MDM2 small-molecule antagonist RG7112 activates p53 signaling and regresses human tumors in preclinical cancer models. Cancer Res. 73, 2587-2597 (2013).

39. Lengner, C. J. et al. Osteoblast differentiation and skeletal development are regulated by Mdm2-p53 signaling. J. Cell Biol. 172, 909-921 (2006).

40. Walton, M. I., Wilson, S. C., Hardcastle, I. R., Mirza, A. R. \& Workman, P. An evaluation of the ability of pifithrin-alpha and -beta to inhibit p53 function in two wild-type p53 human tumor cell lines. Mol. Cancer Ther. 4, 1369-1377 (2005).

41. Kim, B. G., Lee, J. H., Yasuda, J., Ryoo, H. M. \& Cho, J. Y. Phospho-Smad1 modulation by nedd4 E3 ligase in BMP/TGF-beta signaling. J. Bone. Miner. Res. 26, 1411-1424 (2011).

42. Kulikov, R. et al. Mdm2 facilitates the association of p53 with the proteasome Proc. Natl Acad. Sci. USA 107, 10038-10043 (2010).

43. Carroll, P. E. et al. Centrosome hyperamplification in human cancer: chromosome instability induced by $\mathrm{p} 53$ mutation and/or Mdm2 overexpression. Oncogene 18, 1935-1944 (1999).

44. Oliner, J. D., Kinzler, K. W., Meltzer, P. S., George, D. L. \& Vogelstein, B. Amplification of a gene encoding a p53-associated protein in human sarcomas. Nature 358, 80-83 (1992).

45. Schroeder, T. M. \& Westendorf, J. J. Histone deacetylase inhibitors promote osteoblast maturation. J. Bone Miner. Res. 20, 2254-2263 (2005).

46. Lee, H. W. et al. Histone deacetylase 1-mediated histone modification regulates osteoblast differentiation. Mol. Endocrinol. 20, 2432-2443 (2006).

47. Ducy, P., Zhang, R., Geoffroy, V., Ridall, A. L. \& Karsenty, G. Osf2/Cbfa1: a transcriptional activator of osteoblast differentiation. Cell 89, 747-754 (1997).

48. Kook, H. et al. Cardiac hypertrophy and histone deacetylase-dependent transcriptional repression mediated by the atypical homeodomain protein Hop. J. Clin. Invest. 112, 863-871 (2003).

49. Jessberger, S. et al. Epigenetic modulation of seizure-induced neurogenesis and cognitive decline. J. Neurosci. 27, 5967-5975 (2007).

50. Rao, R. S., Miano, J. M., Olson, E. N. \& Seidel, C. L. The A10 cell line: a model for neonatal, neointimal, or differentiated vascular smooth muscle cells? Cardiovasc. Res. 36, 118-126 (1997).

51. Kimes, B. W. \& Brandt, B. L. Characterization of two putative smooth muscle cell lines from rat thoracic aorta. Exp. Cell Res. 98, 349-366 (1976). 
52. Phan, O. et al. Sevelamer prevents uremia-enhanced atherosclerosis progression in apolipoprotein E-deficient mice. Circulation 112, 2875-2882 (2005).

53. Lee, K. et al. Targeting of the osteoclastogenic RANKL-RANK axis prevents osteoporotic bone loss and soft tissue calcification in coxsackievirus B3-infected mice. J. Immunol. 190, 1623-1630 (2013).

54. Price, P. A., Faus, S. A. \& Williamson, M. K. Warfarin-induced artery calcification is accelerated by growth and vitamin D. Arterioscler. Thromb. Vasc. Biol. 20, 317-327 (2000).

55. Price, P. A., June, H. H., Buckley, J. R. \& Williamson, M. K. Osteoprotegerin inhibits artery calcification induced by warfarin and by vitamin D. Arterioscler. Thromb. Vasc. Biol. 21, 1610-1616 (2001).

56. Kang, W. S. et al. A macrophage-specific synthetic promoter for therapeutic application of adiponectin. Gene Ther. 21, 353-362 (2014).

57. Nam, D. et al. Partial carotid ligation is a model of acutely induced disturbed flow, leading to rapid endothelial dysfunction and atherosclerosis. Am. J. Physiol. Heart Circ. Physiol. 297, H1535-H1543 (2009).

58. Potthoff, M. J. et al. Histone deacetylase degradation and MEF2 activation promote the formation of slow-twitch myofibers. J. Clin. Invest. 117, 2459-2467 (2007).

59. Ye, F., Lattif, A. A., Xie, J., Weinberg, A. \& Gao, S. Nutlin-3 induces apoptosis, disrupts viral latency and inhibits expression of angiopoietin-2 in Kaposi sarcoma tumor cells. Cell Cycle 11, 1393-1399 (2012).

60. Montgomery, R. L. et al. Histone deacetylases 1 and 2 redundantly regulate cardiac morphogenesis, growth, and contractility. Genes Dev. 21, 1790-1802 (2007).

61. Haberland, M., Johnson, A., Mokalled, M. H., Montgomery, R. L. \& Olson, E. N. Genetic dissection of histone deacetylase requirement in tumor cells. Proc. Natl Acad. Sci. USA 106, 7751-7755 (2009).

62. Eom, G. H. et al. Regulation of acetylation of histone deacetylase 2 by p300/CBP-Associated factor/histone deacetylase 5 in the development of cardiac hypertrophy. Circ. Res. 114, 1133-1143 (2014).

\section{Acknowledgements}

We are grateful to Professors Eric N. Olson and Rhonda Bassel-Duby at the University of Texas Southwestern Medical Center for providing HDAC1 conditional KO mice and for critically reviewing the manuscript; to Professor Jonathan A. Epstein for critical comments on the manuscript; to Professors Je-Yeol Cho (Seoul National University) and Young-Kyu Koh (Korea University) for their help with the ubiquitination assay and for critical reading; and to Professor Dae-Won Chung for the calcification assay (Yeung-Nam University). This research was supported by Basic Science Research Program through the National Research Foundation of Korea funded by the Ministry of Education (2015R1A2A1A05001708), by a National Research Foundation of Korea grant
(MRC, 2011-0030132) funded by the Korean government (MSIP), and by a grant of the Korean Health Technology R\&D Project, Ministry of Health \& Welfare, Republic of Korea (A121260 and A111345).

\section{Author contributions}

D.-H.K., G.H.E. and J.H.K. performed most experiments. S.S. prepared VSMCs and performed the measurements of calcium content. H.-K.M., W.K, Y.S.N. and E.R.W. performed the animal experiments. H.J. performed the immunoprecipitation and ubiquitination assays. T.K. and N.C. performed the qRT-PCR analysis. S.Y. and K.-I.N performed all histological analyses. I.-K.L. and H.S.K. diagnosed and prepared human samples. H.C. and J.-T.K. prepared and performed microcomputed tomography. D.H.P., K.S. and N.K. provided critical protocols and tips for many experimental procedures. Y.S.K. and Y.A. performed the carotid artery ligation experiment with ApoE KO mice. H.-J.C. performed the high calcium plus high-cholesterol diet experiment with ApoE KO mice. I.-K.L. provided high-cholesterol-administered ApoE $\mathrm{KO}$ mice and helped virus preparation. H.S.K., J.-T.K., N.K., Y.A., H.-J.C., I.-K.L., K.-I.N., D.H.P., K.S and S.M.M. discussed the results and gave critical comments on the manuscript. H.K. designed the research and provided funding. D.-H.K., G.H.E. and H.K. wrote the manuscript.

\section{Additional information}

cDNA microarray data deposition: Gene expression data have been deposited in the GEO database under accession code GSE74755.

Supplementary Information accompanies this paper at http://www.nature.com/ naturecommunications

Competing financial interests: The authors declare no competing financial interests.

Reprints and permission information is available online at http://npg.nature.com/ reprintsandpermissions/

How to cite this article: Kwon, D.-H. et al. MDM2 E3 ligase-mediated ubiquitination and degradation of HDAC1 in vascular calcification. Nat. Commun. 7:10492 doi: $10.1038 /$ ncomms10492 (2016).

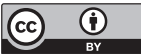

This work is licensed under a Creative Commons Attribution 4.0 International License. The images or other third party material in this article are included in the article's Creative Commons license, unless indicated otherwise in the credit line; if the material is not included under the Creative Commons license, users will need to obtain permission from the license holder to reproduce the material. To view a copy of this license, visit http://creativecommons.org/licenses/by/4.0/ 